Preprint typeset using $\mathrm{AT}_{\mathrm{E}} \mathrm{X}$ style emulateapj v. 5/2/11

\title{
DWARF GALAXY FORMATION WITH H2-REGULATED STAR FORMATION
}

\author{
Michael Kuhlen \\ Theoretical Astrophysics Center, University of California, Berkeley, CA 94720 \\ Mark R. Krumholz \\ Department of Astronomy \& Astrophysics, University of California, Santa Cruz, CA 95064 \\ Piero MADAU \\ Department of Astronomy \& Astrophysics, University of California, Santa Cruz, CA 95064 \\ BRITTON D. SMith \\ Department of Physics and Astronomy, Michigan State University, East Lansing, MI 48824
}

JOHN WISE ${ }^{\dagger}$

Department of Astrophysical Sciences, Princeton University, Peyton Hall, Ivy Lane, Princeton, NJ 08544 Center for Relativistic Astrophysics, Georgia Institute of Technology, 837 State Street, Atlanta, GA 30332

\begin{abstract}
We describe cosmological galaxy formation simulations with the adaptive mesh refinement code Enzo that incorporate a star formation prescription regulated by the local abundance of molecular hydrogen. We show that this $\mathrm{H}_{2}$-regulated prescription leads to a suppression of star formation in low mass halos $\left(M_{\mathrm{h}} \lesssim 10^{10} \mathrm{M}_{\odot}\right)$ at $z>4$, alleviating some of the dwarf galaxy problems faced by theoretical galaxy formation models. $\mathrm{H}_{2}$ regulation modifies the efficiency of star formation of cold gas directly, rather than indirectly reducing the cold gas content with "supernova feedback". We determine the local $\mathrm{H}_{2}$ abundance in our most refined grid cells (76 proper parsec in size at $\left.z=4\right)$ by applying the model of Krumholz, McKee, \& Tumlinson, which is based on idealized 1D radiative transfer calculations of $\mathrm{H}_{2}$ formation-dissociation balance in $\sim 100 \mathrm{pc}$ atomic-molecular complexes. Our $\mathrm{H}_{2}$-regulated simulations are able to reproduce the empirical (albeit lower $z$ ) Kennicutt-Schmidt relation, including the low $\Sigma_{\text {gas }}$ cutoff due to the transition from atomic to molecular phase and the metallicity dependence thereof, without the use of an explicit density threshold in our star formation prescription. We compare the evolution of the luminosity function, stellar mass density, and star formation rate density from our simulations to recent observational determinations of the same at $z=4-8$ and find reasonable agreement between the two.
\end{abstract}

Subject headings: cosmology: theory - galaxies: dwarfs - galaxies: formation - galaxies: halos methods: numerical

\section{INTRODUCTION}

The $\Lambda$ CDM paradigm of cosmological structure formation (White \& Rees 1978; Blumenthal et al. 1984) has been tremendously successful at explaining the large scale, statistical features of the distribution of matter in our universe (Springel et al. 2006). At the same time, it is also very clear that the mapping from dark matter halos to their baryonic components, to the properties of galaxies embedded within the halos, is far from straightforward and currently poorly understood. One prominent example of this lack of understanding is the fact that the cosmic mass-to-light relation is neither constant nor monotonic, and instead exhibits a minimum at a galaxy mass of $10^{12} \mathrm{M}_{\odot}$ (e.g. Conroy \& Wechsler 2009). Evidently some unknown processes are inhibiting efticient star formation on both larger and smaller mass scales.

The focus of this paper is on the low mass end, the

mqk@astro.berkeley.edu

THubble Fellow dwarf galaxies. There are (at least) two dwarf galaxy problems, which may or may not have the same explanation. The first of these is the well-known "Missing Satellites Problem" (Kauffmann et al. 1993, Klypin et al. 1999, Moore et al. 1999), which refers to the discrepancy between the relatively small number of satellite galaxies known to be orbiting the Milky Way and M31 ( 20 around each) and the vastly larger number of dark matter subhalo satellites predicted from darkmatter-only cosmological numerical simulations $\left(\gtrsim 10^{5}\right.$ in the latest simulations, Diemand et al. 2008, Stadel et al. 2009; Springel et al. 2008). Photo-heating from the meta-galactic UV background will prevent gas from collapsing and forming stars in all but the most massive subhalos (Efstathiou 1992; Kauffmann et al. 1993, Bullock et al. 2000), but even so the number of dark matter subhalos that should be able to host a luminous component, either because they collapsed prior to reionization or because they reached a sufficiently large mass thereafter, exceeds the current census of dwarf satellite galaxies by at least one order of magnitude (Madau et al. 
2008). The interpretation of this discrepancy is further complicated by interactions with the host galaxy. Ram pressure stripping (Mayer et al.2006), as well as resonant (D'Onghia et al. 2009) and tidal interactions (Gnedin et al. 1999) with the host's dark matter halo or stellar disk could modify the abundance and properties of galactic satellite galaxies.

The second dwarf galaxy problem occurs in the field, and is exemplified by the apparent inability of virtually all theoretical models of galaxy formation to match the abundance of low stellar mass galaxies at $z>0$. Semianalytic galaxy formation models (SAMs), for example, are able to match the observed stellar mass function in the local universe $(z=0)$ by judiciously tuning their AGN, photo-ionization, and supernova feedback parameters, but these same models predict an abundance of $M_{\star}<10^{10} \mathrm{M}_{\odot}$ galaxies at higher redshifts that exceeds the observational constraints by an order of magnitude (Fontanot et al. 2009, Marchesini et al. 2009, Cirasuolo et al. 2010). Hydrodynamic galaxy formation simulations face similar problems (Nagamine et al. 2006; Cen \& Ostriker 2006, Choi \& Nagamine 2011). A closely related problem is the inability of numerical simulations and SAMs to match the low values of the stellar mass fraction and its strongly decreasing trend with halo mass, as inferred from observations and semi-empirical approaches (Guo et al. 2010, Avila-Reese et al. 2011).

Besides UV photo-heating, stellar feedback, in the form of energy or momentum injection from supernovae explosions (Dekel \& Silk 1986, Efstathiou 2000), stellar winds (Norman \& Silk 1980, McKee 1989), or radiation pressure ( et al. 2011), is commonly invoked to explain the reduction in star formation efficiency in low mass halos. In SAMs (Cole et al. 2000, Benson et al. 2003, Somerville et al. 2008; Wang et al. 2008) this type of feedback is typically modeled as a reduction of the cold gas reservoir available for star formation, with an efficiency proportional to some power law of the galactic disk circular velocity.

In direct cosmological numerical simulations, stellar feedback remains subgrid physics even for today's stateof-the-art computational efforts. Cosmological zoom-in simulations of individual galaxies have reached tens of parsec resolution (Gnedin et al. 2009; Ceverino \& Klypin 2009, Governato et al. 2010, Agertz et al. 2011; FaucherGiguère \& Kereš 2011; Guedes et al.|2011), but full-box global simulations are at least one order of magnitude behind (Ocvirk et al. 2008, Schaye et al. 2010, Oppenheimer et al. 2010, Choi \& Nagamine 2011; FaucherGiguère et al. 2011). Neither approach is able to resolve the $\lesssim$ parsec scales on which stellar feedback actually operates in nature. Instead simulators have turned to feedback prescriptions that are meant to capture the cumulative effect of supernovae explosions on scales that are computationally accessible. A wide variety of such subgrid physics prescriptions have been implemented, ranging from a simple injection of thermal energy at the location of newly created star particles Cen \& Ostriker 1993), often with radiative cooling artificially turned off for some time to prevent the newly added energy from rapidly radiating away (Thacker \& Couchman 2000; Stinson et al. 2006), to attempts at keeping track of separate cold and hot phases of the subgrid interstellar medium
(Yepes et al. 1997; Gnedin 1998, Springel \& Hernquist 2003), to direct kinetic feedback, in which momentum kicks are applied to surrounding gas particles (Springel \& Hernquist 2003; Oppenheimer \& Davé 2006 Schaye et al. 2010; Genel et al. 2010), which are subsequently temporarily decoupled from hydrodynamic forces (except in Schaye et al. 2010) in order to allow them to escape the star forming region.

Although improvements in the stellar feedback treatment have indeed enabled progress in galaxy formation simulations, for example the production of quasi-realistic disk galaxies from cosmological initial conditions (Governato et al. 2010; Agertz et al. 2011; Guedes et al. 2011; Avila-Reese et al.2011; Piontek \& Steinmetz 2011; Brook et al. 2012), the results depend sensitively on the details of the feedback implementations (Sales et al. 2010), which themselves are often based on ad-hoc assumptions. Furthermore, many problems are not completely solved by stellar feedback as it is currently implemented. The inability of simulations and SAMs, even those including supernova feedback prescriptions, to match the observed high redshift stellar mass functions and star formation rates is one example (Cirasuolo et al. 2010, Choi \& Nagamine 2011). Another is the challenge of suppressing the stellar mass content of low mass halos while simultaneously matching the observed mass-metallicity relation in Milky Way dwarf satellites (Font et al. 2011).

Important physical processes associated with star formation are not captured by current models, and it is time to revisit the dwarf galaxy problems in light of new understanding of how SF actually occurs in the ISM of local galaxies. One promising direction is an improved treatment of the chemistry and thermodynamics of the interstellar gas that is actually forming stars. In particular, spatially resolved observations of local galaxies have revealed that star formation correlates much more tightly with the density of molecular gas than total gas density (Wong \& Blitz 2002; Kennicutt et al.|2007; Leroy et al. 2008, Bigiel et al. 2008). Even though the primary cooling agents are lines of $\mathrm{CO}$ or CII (depending on the chemical state of the carbon), molecular hydrogen $\left(\mathrm{H}_{2}\right)$ is expected to be good tracer of star formation, even at low metallicities (Krumholz et al. 2011).

This motivates a star formation prescription that differentiates between the chemical phases of the gas, in which the formation of star particles is tied to the local abundance of $\mathrm{H}_{2}$, as opposed to the total gas density, as is more commonly done in numerical simulations. Indeed some SAMs have begun to explore this direction (Fu et al. 2010, del P. Lagos et al. 2010, Krumholz \& Dekel 2011), and several numerical simulations including $\mathrm{H}_{2}$ physics have been published (Robertson \& Kravtsov 2008; Gnedin et al. 2009, Feldmann et al. 2011). Until now these simulations focused only on individual galaxies, either in isolated disks or in cosmological zoom-in simulations. In this work we investigate for the first time the effect of an $\mathrm{H}_{2}$-regulated star formation prescription in full-box cosmological simulations, with an eye towards the statistical distribution of star formation efficiency in dwarf galaxies.

Following the non-equilibrium $\mathrm{H}_{2}$ chemistry in a realistic and self-consistent manner, including formation on dust grains and the radiative transfer of ionizing and dissociating radiation, is complicated and expen- 
sive to implement in numerical galaxy formation simulations (Gnedin et al. 2009). Fortunately, analytical 1D radiative transfer calculations assuming $\mathrm{H}_{2}$ formationdissociation balance (Krumholz et al. 2008, 2009, McKee \& Krumholz 2010) have shown that the $\mathrm{H}_{2}$ abundance is determined to a good approximation (Krumholz \& Gnedin 2011) by the HI column density and metallicity of gas on $\sim 100 \mathrm{pc}$ scales. As these scales are directly accessible to us, we can bypass much of the computational difficulty associated with proper $\mathrm{H}_{2}$ chemistry by implementing the Krumholz et al. (2009) results in our cosmological simulations. Even so, our use of cosmological adaptive mesh refinement prevents our simulations from progressing much past $z \sim 4$ at an acceptable computational expense. Yet it is precisely in the early universe, at low but non-zero metallicities, that the metallicitydependence of the $\mathrm{HI}$ to $\mathrm{H}_{2}$ transition will be most important, and its effect on star formation greatest. Thanks to extensive multi-wavelength surveys (e.g. the Great Observatories Origins Deep Survey, Giavalisco et al. 2004) and deep follow-up observations with the Hubble and Spitzer space telescopes (e.g. Stark et al. 2009, Bouwens et al. 2011; González et al. 2011; Labbé et al. 2010a), we are able to make contact with observational constraints on the cosmic stellar mass and star formation density even at these high redshifts.

The main driver of this work, then, is to investigate to what degree a proper accounting of the $\mathrm{H}_{2}$ abundance in star forming gas can take the role that is traditionally assigned to supernova feedback, namely a reduction of the star formation efficiency in low mass dark matter halos (see also Gnedin et al. 2009, Gnedin \& Kravtsov 2010. hereafter G09; GK10). This paper is organized as follows. In $\S 2$ we describe our numerical approach and the details of our $\mathrm{H}_{2}$-regulated star formation prescription. We present the results of our work in $\S 3-5$. We first show that our prescription reproduces many of the observational features of the star formation scaling relations $(\S 3$. We then demonstrate that tying star formation to the $\mathrm{H}_{2}$ abundance indeed suppresses star formation in low mass halos, thereby alleviating the dwarf galaxy problems $(\S$ 4). Finally, we present a direct comparison to recent observational determinations of the high redshift evolution of the luminosity function, stellar mass density, and star formation rate density ( $\S 5$. We summarize and conclude in $\S 6$

\section{SIMULATIONS}

We have conducted cosmological AMR hydrodynamics simulations using Enzo v2. [1 to follow galaxy formation in the early $(z \geq 4)$ universe. The computational domain covers a $(12.5 \mathrm{Mpc})^{3}$ box with a root grid of $256^{3}$ grid cells. The dark matter density field is resolved with $256^{3}$ particles of mass $3.1 \times 10^{6} \mathrm{M}_{\odot}$. The box has mean over-density of zero, and no additional density fluctuation on the scale of the simulation box ("DC mode", Gnedin et al. 2011) has been applied. Adaptive mesh refinement is allowed to occur throughout the entire domain for a maximum of 7 levels of refinement, resulting in a maximum spatial resolution of $\Delta x_{7}=76.3 \times 5 /(1+z)$ proper parsec. Mesh refinement is triggered by a grid cell reaching either a dark matter mass equal to 4 times

\footnotetext{
${ }^{1}$ http://code.google.com/p/enzo/
}

the mean root grid cell dark matter mass, or a baryonic mass equal to $8 \times 2^{-0.4 l}$ times the mean root grid cell baryonic mass, where $l$ is the grid level. The negative exponent in the baryonic refinement mass threshold implies a super-Lagrangian refinement criterion that results in more aggressive refinement at higher resolution. The simulations are initialized at $z_{i}=99$ with the Eisenstein $\& \mathrm{Hu}(1999)$ transfer function, and cosmological parameters consistent with the WMAP 7-year results (Komatsu et al. 2011): $\Omega_{M}=0.265, \Omega_{\Lambda}=0.735, \Omega_{b} h^{2}=0.02264$, $h=0.71, n=0.963$, and $\sigma_{8}=0.801$. The parameters of our suite of simulations are summarized in Table 1.

The equations of hydrodynamics are solved using Enzo's implementation of the Piecewise Parabolic Method (PPM, Colella \& Woodward 1984), a higher order accurate Godunov scheme. We utilize the recently added HLLC Riemann solver (Toro et al. 1994) with a fallback scheme to the more diffusive HLL solver for problematic cells, which greatly aids the simulations' stability. Enzo employs a dual-energy formalism (Bryan et al. 1995), solving for both the internal gas energy and total energy separately, to ensure accurate pressures and temperatures in hypersonic flows.

Our simulations include radiative cooling from both primordial and metal enriched gas, as well as photoheating from an optically thin, uniform meta-galactic UV background. The primordial gas cooling rates are calculated from the ionization states of hydrogen and helium, which are followed with a 6 -species $\left(\mathrm{H}, \mathrm{H}^{+}, \mathrm{He}, \mathrm{He}^{+}\right.$, $\mathrm{He}^{++}$, and $\mathrm{e}^{-}$) non-equilibrium chemical network (Abel et al. 1997; Anninos et al. 1997), including collisional and photo-ionization/excitation rates. The metal cooling is determined from a 5-dimensional table (independent variables: density, temperature, electron fraction, metallicity, and redshift) of heating and cooling rates precomputed with the Cloudy code (Ferland et al. 1998), as described in detail in Smith et al. (2008) and Smith et al. (2011). For the UV background we used the updated version of the Haardt \& Madau (2001) UV background model that ships with version 07.02.01 of Cloudy. This model includes the contributions of both quasars and galaxies in a redshift dependent manner, and is in reasonable agreement with a more recent calculation of the cosmic UV background (Faucher-Giguère et al.|2009).

As is commonly done in Eulerian hydrodynamic galaxy formation simulations (Machacek et al. 2001; Robertson \& Kravtsov 2008, Agertz et al. 2009, Ceverino \& Klypin 2009), we apply an artificial pressure support to cells that have reached the maximum refinement level. This is necessary in order to stabilize these cells against artificial fragmentation, and is supposed to mimic the pressure support from turbulent motions below the simulation's resolution. In Enzo this support is implemented by increasing the internal gas energy up to some multiple of the value required to make the cell Jeans stable. We have set this factor equal to 10 , meaning that the Jeans length of the highest resolution cells is artificially set to $\sqrt{10} \approx 3$ times the cell width.

\subsection{Star Formation Prescriptions}

The star formation (hereafter SF) prescriptions we have implemented are all variations on the basic Schmidt law, whereby the local star formation rate (hereafter 
Table 1

Summary of the simulations.

\begin{tabular}{|c|c|c|c|c|c|c|}
\hline Name & $z_{\text {final }}$ & $\rho_{\text {gas, } \mathrm{SF}}$ & $n_{\text {thresh }}$ & $J_{\mathrm{LW}} / J_{\mathrm{MW}}$ & {$\left[\mathrm{Z}_{\text {floor }}\right]$} & Comment \\
\hline KT07 & 4.0 & tot & $50 \mathrm{~cm}^{-3}$ & - & - & Krumholz \& Tan (2007) SF law \\
\hline KT07_low & 6.0 & tot & $5 \mathrm{~cm}^{-3}$ & - & - & lower SF threshold \\
\hline KT07_high & 6.0 & tot & $500 \mathrm{~cm}^{-3}$ & - & - & higher SF threshold \\
\hline КМT09 & 4.0 & $\mathrm{H}_{2}$ & - & - & -3.0 & Krumholz et al. (2009): 2-phase equilibrium \\
\hline KMT09_L8 & 6.0 & $\mathrm{H}_{2}$ & - & - & -3.0 & one additional refinement level (maxlevel $=8$ ) \\
\hline KMT09_FLW1 & 5.0 & $\mathrm{H}_{2}$ & - & 1 & -3.0 & KMT09 with uniform LW \\
\hline KMT09_FLW10 & 5.0 & $\mathrm{H}_{2}$ & - & 10 & -3.0 & background of \\
\hline KMT09_FLW100 & 5.0 & $\mathrm{H}_{2}$ & - & 100 & -3.0 & increasing \\
\hline KMT09_FLW1000 & 5.0 & $\mathrm{H}_{2}$ & - & 1000 & -3.0 & intensity \\
\hline KMT09_ZF4.0 & 6.0 & $\mathrm{H}_{2}$ & - & - & -4.0 & lower $\mathrm{Z}_{\text {floor }}$ \\
\hline KMT09_ZF2.5 & 6.0 & $\mathrm{H}_{2}$ & - & - & -2.5 & higher $\mathrm{Z}_{\text {floor }}$ \\
\hline KMT09_ZF2.0 & 6.0 & $\mathrm{H}_{2}$ & - & - & -2.0 & even higher $Z_{\text {floor }}$ \\
\hline KMT09_ZFz10 & 6.0 & $\mathrm{H}_{2}$ & - & - & -3.0 & $\mathrm{Z}_{\text {floor }}$ at $z=10$ \\
\hline KMT09_Sob & 5.0 & $\mathrm{H}_{2}$ & - & - & -3.0 & Sobolev-like approximation of $\Sigma$ \\
\hline KMT09_SobL8 & 6.0 & $\mathrm{H}_{2}$ & - & - & -3.0 & KMT09_Sob with maxlevel=8 \\
\hline
\end{tabular}

Note. - All simulations have the same box size $(12.5 \mathrm{Mpc})$ and were initialized at $z=99$ with a WMAP7 cosmology: $\Omega_{M}=0.265, \Omega_{\Lambda}=0.735, \Omega_{b}=0.045, h=0.71, \sigma_{8}=0.801$, and $n_{s}=0.963$. The number of dark matter particles is $256^{3}\left(m_{p}=3.64 \times 10^{6} \mathrm{M} \odot\right)$, and the root grid dimensions are also $256^{3}$. We allow up to 7 levels of adaptive mesh refinement, except in KMT09_L8 which has one additional level. $\rho_{\text {gas,SF }}$ indicates whether the SF prescription is tied to the total or $\mathrm{H}_{2}$ gas density, $n_{\text {thresh }}$ is the minimum density required for SF to occur (KT07 runs only), $J_{\mathrm{LW}} / J_{\mathrm{MW}}$ the intensity of the Lyman-Werner background normalized to the Milky Way's value (KMT09_FLW runs only), and $\left[\mathrm{Z}_{\text {floor }}\right] \equiv \log _{10}\left(\mathrm{Z}_{\mathrm{floor}} / \mathrm{Z}_{\odot}\right)$ is the initial seed metallicity applied at $z=9$ in the KMT09 simulations.

SFR) in a grid cell is proportional to its gas density divided by a $\mathrm{SF}$ time scale,

$$
\dot{\rho}_{\mathrm{SF}}=\epsilon \frac{\rho_{\mathrm{gas}}}{t_{\star}} .
$$

In all of our simulations we set the SF time scale equal to the local free-fall time,

$$
t_{\star}=t_{\mathrm{ff}}=\sqrt{\frac{3 \pi}{32 G \rho_{\mathrm{gas}}}},
$$

and fix the SF efficiency to $\epsilon=0.01$, as motivated by Krumholz \& Tan (2007), who showed that the local SF efficiency per free fall time is low $\left(\epsilon_{\mathrm{SF}} \equiv\right.$ $\left.\Sigma_{\mathrm{SFR}} /\left(\Sigma_{\text {gas }} t_{\mathrm{ff}}\right) \approx 0.01\right)$ and approximately constant over 4 orders of magnitude in density.

In Enzo's standard routines, SF is allowed to occur at every time step and in every grid cell that is not further refined. Provided the cell fulfills all conditions for SF, a fraction of the cell's gas mass is converted into a star particle of mass $m_{p}=\epsilon \rho_{\text {gas }}(\Delta x)^{3}\left(\Delta t / t_{\star}\right)$. For highly refined cells with small time steps $\Delta t$, this commonly result in very large numbers of low mass star particles, which can dramatically slow down the simulation's progress. Applying a mass threshold, below which a star particle is simply not created, is undesirable, since it can lead to a significant amount of "unfulfilled" SF, although this can be remedied with a stochastic SF criterion (e.g. Springel \& Hernquist 2003, and see below).

To overcome these difficulties, we have modified Enzo's routines to allow SF to occur only once per root grid time step and only in cells at the highest refinement level (here $l=7$ ), but with a star particle mass proportional to the root grid time step $\Delta t_{0}$ (see Kravtsov 2003), i.e.

$$
m_{p}=\epsilon \rho_{\text {gas }}\left(\Delta x_{7}\right)^{3} \frac{\Delta t_{0}}{t_{\star}} .
$$

Making $m_{p}$ proportional to the root grid time step $\left(\Delta t_{0} \gg \Delta t_{7}\right)$ goes a long way towards overcoming the problem of large numbers of low mass star particles. Nevertheless we also enforce a minimum star particle mass of $m_{\text {min }}=10^{4} \mathrm{M}_{\odot}$, since even $\Delta t_{0}$ can occasionally become very small. Below this mass we implement a stochastic $\mathrm{SF}$ criterion as follows: if $m_{p}<m_{\mathrm{min}}$, we form a particle of mass equal to $m_{\min }$ if a randomly generated number is smaller than $\left(m_{p} / m_{\min }\right)$.

We consider two distinct classes of SF prescriptions:

i) Standard SFR (KT0\%): The SFR is proportional to the total gas density divided by the free-fall time, resulting in a SFR proportional to $\rho_{\text {gas }}^{3 / 2}$. We apply a density threshold below which $\mathrm{SF}$ is not allowed to occur, and vary this threshold between values of 5 , 50 , and $500 \mathrm{~cm}^{-3}$.

ii) $H_{2}$-regulated $S F R$ (KMT09): The SFR is proportional to the molecular hydrogen density divided by the free-fall time determined from the total gas density, resulting in a SFR proportional to $f_{\mathrm{H}_{2}} \rho_{\text {gas }}^{3 / 2}$. The $\mathrm{H}_{2}$ fraction, $f_{\mathrm{H}_{2}}=\rho_{\mathrm{H}_{2}} / \rho_{\text {gas }}$, is determined following Krumholz et al. (2009) (more details in section 2.2), and we consider both the two-phase equilibrium model and a range of models with different Lyman-Werner $\mathrm{H}_{2}$-dissociating background intensities. No density threshold is applied.

The resolution of our simulations is not sufficient to resolve the formation sites of the first generation of stars, the so-called population III. In order to capture the metal enrichment resulting from the supernova explosions of this primordial stellar population, we instantaneously introduce a metallicity floor of $\left[\mathrm{Z}_{\text {floor }}\right] \equiv \log _{10}\left(\mathrm{Z}_{\text {floor }} / \mathrm{Z}_{\odot}\right)=$ -3.0 at $z=9$, as motivated by recent high resolution numerical simulations of the transition from Pop.III to Pop.II SF (Wise et al. 2012). This ensures the presence of a minimum amount of metals, which seed subsequent star formation and further metal enrichment. We discuss the sensitivity of our results to the time and amplitude of this metallicity floor in $\S 4.3$. 


\subsection{Molecular Chemistry}

To obtain the molecular hydrogen mass fraction $f_{\mathrm{H}_{2}} \equiv$ $\rho_{\mathrm{H}_{2}} / \rho_{\text {gas }}$ in a given grid cell, we follow the analytical model developed in Krumholz et al. (2008), Krumholz et al. (2009), and McKee \& Krumholz (2010). This model is based on a radiative transfer calculation of an idealized spherical giant atomic-molecular complex, subject to a uniform and isotropic Lyman-Werner (LW) radiation field. The $\mathrm{H}_{2}$ abundance is calculated assuming formation-dissociation balance. The solution of this problem can conveniently be expressed in three lines:

$$
\begin{gathered}
f_{\mathrm{H}_{2}} \simeq 1-\frac{3}{4} \frac{s}{1+0.25 s}, \\
s=\frac{\ln \left(1+0.6 \chi+0.01 \chi^{2}\right)}{0.6 \tau_{c}}, \\
\chi=71\left(\frac{\sigma_{d,-21}}{\mathcal{R}_{-16.5}}\right) \frac{G_{0}^{\prime}}{n_{\mathrm{H}, 0}},
\end{gathered}
$$

where $\tau_{c}$ is the dust optical depth of the cloud, $\sigma_{d,-21}$ is the dust cross-section per $\mathrm{H}$ nucleus to $1000 \AA$ radiation, normalized to a value of $10^{-21} \mathrm{~cm}^{-2}, \mathcal{R}_{-16.5}$ is the rate coefficient for $\mathrm{H}_{2}$ formation on dust grains, normalized to the Milky Way value of $10^{-16.5} \mathrm{~cm}^{3} \mathrm{~s}^{-1}$ (Wolfire et al. 2008), $G_{0}^{\prime}$ is the ambient UV radiation field intensity, normalized to the Draine (1978) value for the Milky Way, and $n_{\mathrm{H}, 0}$ is the volume density of $\mathrm{H}$ nuclei in units of $\mathrm{cm}^{-3}$. Since both $\sigma_{d}$ and $\mathcal{R}$ are linearly proportional to the dust abundance, their ratio is independent of the gas metallicity.

Krumholz et al. (2009) showed that a further simplification to the model can be made if the ISM is assumed to be in two-phase equilibrium between a cold neutral medium (CNM) and and a warm neutral medium (WNM) (Wolfire et al. 2003). The assumption of pressure balance between these two ISM components forces the minimum CNM density to be linearly proportional to the intensity of the LW radiation field, with only a weak dependence on metallicity:

$$
n_{\min } \approx \frac{31}{1+3.1\left(\mathrm{Z} / \mathrm{Z}_{\mathrm{SN}}\right)^{0.365}} G_{0}^{\prime},
$$

where $Z_{S N}$ is the gas phase metallicity in the solar neighborhood, and we set $\mathrm{Z}_{\mathrm{SN}}=\mathrm{Z}_{\odot}$ (Rodríguez \& DelgadoInglada 2011) and $\mathrm{Z}_{\odot}=0.0204$. Allowing for the typical CNM density to be somewhat higher than this minimum value, $n=\phi_{\mathrm{CNM}} n_{\min }$, we get

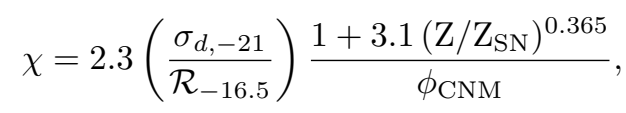

which renders $f_{\mathrm{H}_{2}}$ completely independent of the LW intensity. As in Krumholz \& Gnedin (2011) we set $\phi_{\mathrm{CNM}}=3$ and $\left(\sigma_{d,-21} / \mathcal{R}_{-16.5}\right)=1$.

We have conducted one simulation with this two-phase equilibrium assumption (KMT09), and a range of simulations without it, for which we instead specify a spatially uniform LW background intensity equal to 1, 10, 100, and 1000 times the present day Milky Way value of $7.5 \times 10^{-4}$ LW photons $\mathrm{cm}^{-3}$ (Draine 1978) (KMT09_FLW1, ..., KMT09_FLW1000). We directly apply these prescriptions to the highest resolution grid cells in our simula- tions, whose size $\left(\Delta x_{7}=54.5 \times 7 /(1+z)\right.$ proper par$\mathrm{sec}$ ) is comparable to the physical dimensions of giant atomic-molecular complexes (Blitz 1993). The dust optical depth is given by

$$
\begin{aligned}
\tau_{c} & =\Sigma / \mu_{H} \sigma_{d} \\
& \simeq 0.067\left(\frac{\mathrm{Z}}{\mathrm{Z}_{\mathrm{SN}}}\right)\left(\frac{\rho \Delta x_{7}}{1 \mathrm{M}_{\odot} \mathrm{pc}^{-2}}\right),
\end{aligned}
$$

where $\Sigma=\rho \Delta x_{7}$ is the cell's column density, $\mu_{\mathrm{H}}=$ $2.3 \times 10^{-24} \mathrm{~g}$ is the mean mass per $\mathrm{H}$ nucleus, and we have set the dust cross section per $\mathrm{H}$ nucleus to be $\sigma_{d}=10^{-21}\left(\mathrm{Z} / \mathrm{Z}_{\mathrm{SN}}\right) \mathrm{cm}^{2}$.

The use of $\Delta x_{7}$ in the calculation of $\Sigma$ introduces an undesirable explicit resolution dependence in our algorithm, the effect of which we investigate in $\S 3.4$ and 4.2 . A Sobolev-like approximation, $\Sigma_{\text {Sob }} \equiv \rho \times(\rho / \nabla \rho)$ (e.g. G09; Krumholz \& Gnedin 2011), would remove the explicit dependence of the algorithm on the width of the finest grid cells. As discussed in detail in the Appendix, we have conducted additional simulations using $\Sigma_{\text {Sob }}$ in the KMT09 prescription, and these resulted in slightly lower column densities and reduced SF rates. At densities relevant for star formation $\left(n>5 \mathrm{~cm}^{-3}\right)$, the differences between the two approximations of $\Sigma$ are small, less than $20 \%$ in the median with a scatter of 0.26 dex, and well within the range of uncertainty of the parameters of the KMT09 model. The results presented in this paper were obtained with the simpler and computationally less expensive direct cell-based approximation using $\Sigma_{\text {cell }} \equiv \rho \Delta x_{7}$.

Regardless of how the surface density of atomicmolecular complexes is calculated, we should expect some degree of residual resolution dependence in our simulations, since we don't include the necessary stellar feedback physics (see below) that regulates the structure of molecular clouds and provides pressure support against further collapse. The artificial pressure support mentioned above, which we apply in order to avoid spurious fragmentation, is resolution dependent, and hence higher numerical resolution (through additional refinement levels) will always result in further collapse and higher densities in our simulations.

The KMT09 model has recently been tested against numerical simulations that self-consistently follow the formation and destruction of $\mathrm{H}_{2}$ with a nonequilibrium chemical network including time-dependent and spatially-inhomogeneous 3D radiative transfer of UV and ionizing radiation (Krumholz \& Gnedin 2011). The analytical model agrees extremely well with the numerical results whenever the metallicity is around 1 per cent solar or greater. This agreement holds for both "fixed ISM" simulations in which the metallicity and radiation field are kept constant and in cosmological simulations in which the metallicity and radiation field are computed self-consistently. At metallicities below 1 per cent solar, the analytical model overestimates $f_{\mathrm{H}_{2}}$. Note that for the runs without the two-phase equilibrium assumption, we follow Krumholz \& Gnedin (2011) and apply a "clumping factor" of 30 to the $\mathrm{H}_{2}$ formation rate (i.e. $\left.\left(\sigma_{d,-21} / \mathcal{R}_{-16.5}\right)=1 / 30\right)$ to account for unresolved density inhomogeneities below our simulations' resolution limit. We do not use a clumping factor for the two-phase equilibrium model, because the two-phase model is in ef- 
fect a direct estimate of the proper clumping factor. This physically-motivated clumping model obviates the need for an ad-hoc correction.

\subsection{Feedback}

Although we explore in this work to what degree an improved treatment of the ISM's chemical state can replace the need for stellar feedback in regulating star formation, some form of feedback is necessary even in our simulations, if only to enrich the gas with metals that promote the formation of molecular hydrogen. For this purpose we employ a very simple feedback mechanism that is meant to simultaneously account for the mass, metals, and thermal energy deposited by winds from massive stars and core-collapse supernovae. The feedback is applied instantaneously at the time of formation of the star particle, is deposited into the $l=7$ grid cell containing the particle, and consists of the following three components: (i) a fraction $\epsilon_{m}=0.25$ of the star particle's mass is returned as gas, $\Delta m_{\text {tot }}=\epsilon_{m} m_{p}$; (ii) the gas is enriched with a metal yield of $Y=0.02$, $\Delta m_{Z}=\Delta m_{\text {tot }}\left(Y\left(1-Z_{p}\right)+Z_{p}\right)$, where $Z_{p}$ is the metallicity of the star particle and the two terms correspond to newly and previously enriched ejected material, respectively; (iii) the thermal energy of the gas cell is increased by a fraction $\epsilon_{\mathrm{SN}}=10^{-5}$ of the rest-mass energy of the newly formed star particle, $\Delta E=\epsilon_{\mathrm{SN}} m_{p} c^{2}$.

This feedback implementation is commonly applied in cosmological hydrodynamics simulations, but in fact it is known to be insufficiently strong. The problem with this instantaneous and localized feedback is that the thermal energy is applied to grid cells with very high gas density, in which the cooling time is very short. The injected energy is thus almost immediately lost to radiative cooling, and the feedback "fizzles out" (Katz 1992). We acknowledge the shortcoming of our current feedback implementation, but since we are focusing here on the global effects of different star formation prescriptions, we defer attempts at improving the treatment of feedback to future work.

Finally, we caution that nothing in our implementation of molecular chemistry obviates the need to rely on a subgrid model of star formation. Our model should be viewed in the same light as other subgrid models in wide use, e.g. the two-phase model of Springel \& Hernquist (2003) or the blast wave model of Stinson et al. (2006). The main advantage of our new model is that it incorporates an explicit metallicity-dependence, which both observations and theory appear to demand, as we discuss in more detail below.

\subsection{Halo population}

We used the HOP halo finder (Eisenstein \& Hut 1998) to identify gravitationally bound dark matter halos in our simulations. For every halo we determined the halo center (defined as the location of the highest dark matter density), the virial radius and corresponding mass (defined as the radius enclosing $\Delta_{\text {vir }} \approx$ times the background density (Bryan \& Norman 1998)), and the amount of gas (total, HI, HII, $\mathrm{H}_{2}$, Hel, Hell, and metals) contained within the halo.

Fig. 1 shows the cumulative mass function of the simulations at $z=4$, compared to the Sheth \& Tormen

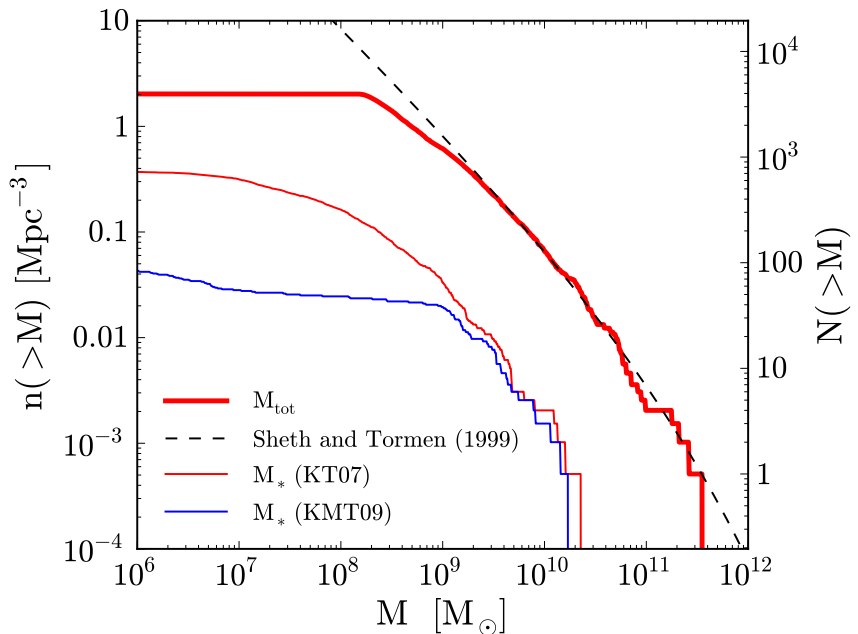

Figure 1. The cumulative mass function of halos in our simulations at $z=4$. The thick line is for total mass (KT07 and KMT09 curves are almost indistinguishable, so we only show KT07), and the thin lines are for the stellar mass. The dashed line shows the Sheth \& Tormen (1999) mass function fit. The right ordinate gives the total number of objects found in our $(12.5 \mathrm{Mpc})^{3}$ simulation volume.

(1999) fit. The agreement is remarkably good down to $M \approx 2 \times 10^{9} \mathrm{M}_{\odot}$, corresponding to halos with $\sim 500$ dark matter particles. At even lower mass numerical resolution effects lead to an artifical suppression in the number of halos. A finer root grid resolution and a more aggressive dark matter based refinement criterion would extend the mass function by another order of magnitude in mass (O'Shea et al. 2005). For our purposes, however, a resolution limit of $\sim 2 \times 10^{9} \mathrm{M}_{\odot}$ is sufficient, since halos with $M<10^{9} \mathrm{M}_{\odot}$ lie below the UV suppression scale . Furthermore, as we show in Sec. 4. $\mathrm{H}_{2}$ regulated SF leads to a suppression of stellar mass in halos with $M \lesssim 10^{10} \mathrm{M}_{\odot}$. As a brief preview of this effect, we overplot in Fig. 1 the cumulative stellar mass functions: the KMT09 simulation has far fewer low stellar mass $\left(\mathrm{M}_{\star}<10^{9} \mathrm{M}_{\odot}\right)$ galaxies, which is a result of the suppression of $\mathrm{SF}$ in halos with $M \lesssim 10^{10} \mathrm{M}_{\odot}$.

\section{THE KENNICUTT-SCHMIDT LAW}

A common test for a new numerical SF implementation is to compare the simulations to the observational Kennicutt-Schmidt (hereafter KS) relation, the empirical power law between the SFR surface density and total gas surface density: $\Sigma_{\mathrm{SFR}} \propto \Sigma_{\text {gas }}^{n}$, with $n \approx 1.4$ (Kennicutt 1998 Daddi et al. 2010, Genzel et al. 2010).

In order to facilitate comparisons to observational data, we determine column densities by integrating through the density fields along the direction perpendicular to the stellar disk of the simulated galaxies. For $\Sigma_{\text {SFR }}$ we integrate a SFR density defined as

$$
\rho_{\mathrm{SFR}}=\sum_{\text {age }<\tau_{\star}} \frac{m_{\star}}{\tau_{\star}(\Delta x)^{3}},
$$

where $\Delta x$ is the cell width, $m_{\star}$ is the star particle mass, and $\tau_{\star}=10 \mathrm{Myr}$ is the SF averaging time scale, which roughly corresponds to observational SFR estimated from nebular emission lines (H $\alpha$, O III) or FIR continuum, but is a factor of $5-10$ shorter than estimates based on FUV measurements (Kennicutt 1998; Feldmann 


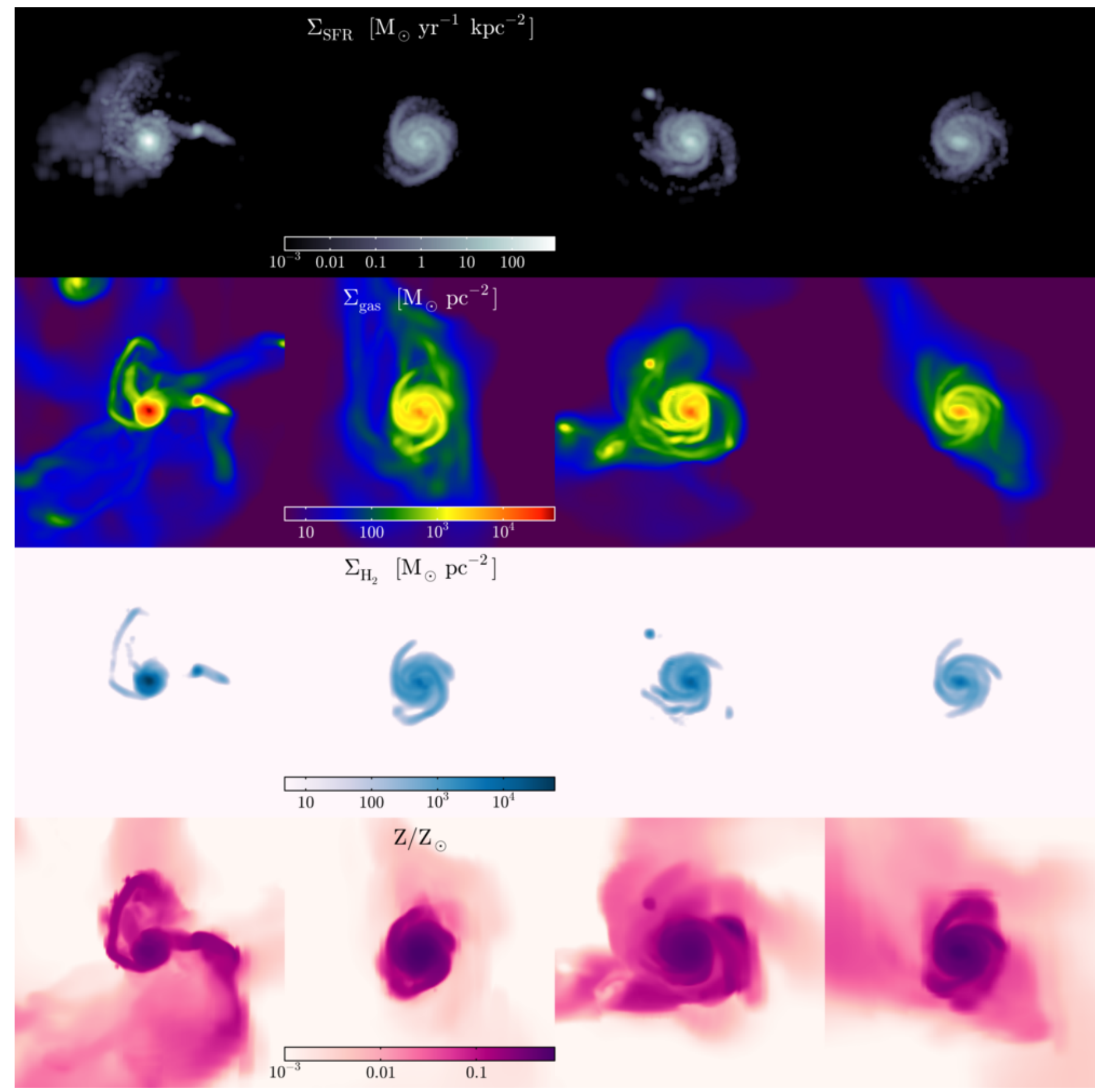

Figure 2. Surface density of SFR, total gas, $\mathrm{H}_{2}$, and metallicity for four representative massive halos $\left(\mathrm{M}_{\mathrm{h}}=2.5 \times 10^{11}, 2.0 \times 10^{11}, 1.6 \times\right.$ $\left.10^{11}, 7.4 \times 10^{10} \mathrm{M}_{\odot}\right)$ in the KMT09 simulation at $z=4$. The projections are calculated as line integrations perpendicular to the disk plane, for a $1000 \times 1000$ pixel grid covering a $10 \times 10 \mathrm{kpc}$ region centered on each galaxy.

et al. 2011). The sum in Equation 10 is over all star particles with age less than $\tau_{\star}$.

We calculate $\Sigma_{\text {gas }}, \Sigma_{\mathrm{H}_{2}}, \Sigma_{\mathrm{HI}}$, and $\Sigma_{\mathrm{SFR}}$ on a $1000 \times$ 1000 pixel grid covering a $10 \times 10 \mathrm{kpc}$ region centered on each galaxy in a sample of 35 of the most massive galaxies in our simulations, chosen to cover a wide range in mean metallicity (see $\S 3.2$. We only considered galaxies in which the adaptive mesh refinement reached the maximum level $(l=7)$. Visualizations of $\Sigma_{\mathrm{SFR}}, \Sigma_{\text {gas }}$, $\Sigma_{\mathrm{H}_{2}}$, and a density-weighted projection of metallicity are shown in Figure 2 for four representative massive galaxies in the KMT09 simulation at $z=4$.

The observational KS relation, as reported for example by Daddi et al. (2010) and Genzel et al. (2010), has been established from a wide variety of data out to $z \gtrsim 3$, including spatially resolved local $z=0$ spiral galaxies, infrared-selected starbursting galaxies and (U)LIRGs, BzK-selected galaxies at $z \approx 1.5$, "normal" star-forming galaxies at $z=1-2.3$, and starbursting sub-millimeter galaxies (SMGs) at $z \approx 1-3$ (see Kennicutt (1998), Daddi et al. (2010), and Genzel et al. (2010) for references). A reproduction of the observational re- 




Figure 3. Comparison of the observational Kennicutt-Schmidt relation from Daddi et al. (2010) (left panel) to a standard SF simulation with SF density threshold of $n_{\text {thresh }}=50 \mathrm{~cm}^{-3}$ (middle) and an $\mathrm{H}_{2}$-regulated SF simulation without any density threshold (right) at $z=4$. In the simulations the surface densities have been determined from line integrations perpendicular to the galaxies' disk plane, and have been smoothed to a resolution of $200 \mathrm{pc}$ (see text for more detail).

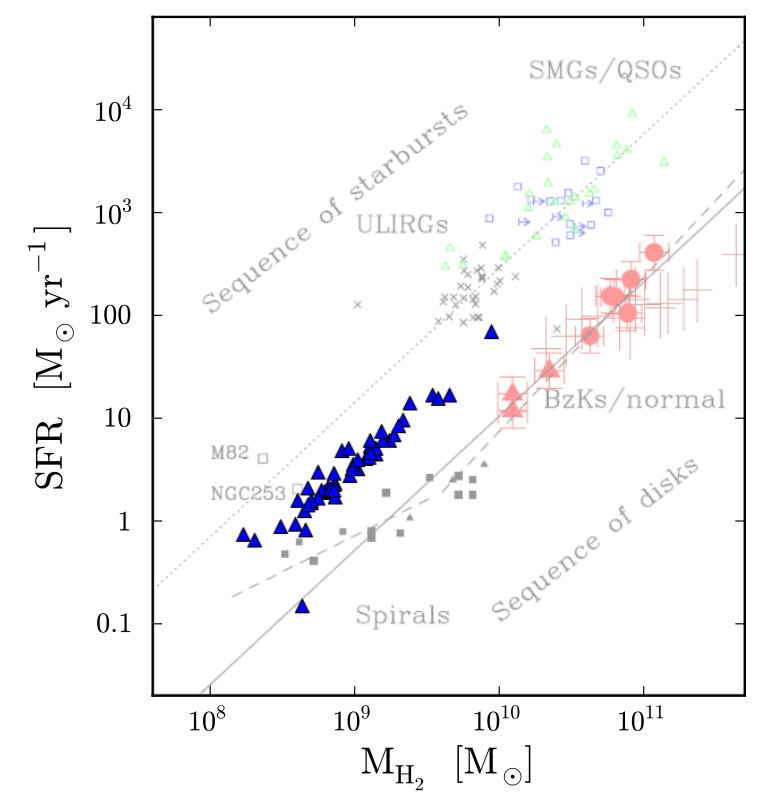

Figure 4. Comparison of the relation between total SFR and total $\mathrm{H}_{2}$ mass for galaxies in the KMT09 simulation at $z=4$ (blue triangles) with the observational results from Daddi et al. (2010) (their Fig.1) for lower redshift galaxies. The simulation and observations have relations with similar slopes, and the simulated galaxies lies in between the "sequence of starbursts" and "sequence of disks". Higher SFR for a given $\mathrm{H}_{2}$ mass may be expected for lower metallicity systems, which require higher densities to allow the transition to $\mathrm{H}_{2}$ to occur.

lation from Daddi et al. (2010) (their Fig.2) is shown in the left panel of Figure 3

Our simulated galaxies are dwarf galaxies with stellar masses less than $10^{10} \mathrm{M}_{\odot}$ at high redshift $z \geq 4$, and hence not directly comparable to any of these observational galaxy samples. Nevertheless, since SF is a local process, it makes sense to directly compare our simulated KS relation to the observations, as a test of how well our
SF prescription is performing. The center and right panels of Figure 3 show this comparison for the KT07 and KMT09 simulations at $z=4$. Since the $10 \mathrm{pc}$ intrinsic resolution of our surface density maps is finer than in most spatially resolved studies to date (e.g. Bigiel et al. 2008: Bolatto et al. 2011), we downgrade the spatial resolution to $200 \mathrm{pc}$ by spatially averaging with a 20 -cell boxcar average. We explore the resolution dependence of our KS relations in $\S 3.3$. Only pixels with non-zero $\Sigma_{\mathrm{SFR}}$ are plotted. The solid line represents a sliding average of $\log _{10} \Sigma_{\text {gas }}$ in $\log _{10} \Sigma_{\mathrm{SFR}}$ bins of width 0.5 .

Both the amplitude and the slope of the relation are qualitatively in very good agreement with the observations. Such agreement has previously been reported (Kravtsov 2003; Gnedin \& Kravtsov 2010, 2011; Feldmann et al. 2011), and we show it here merely to demonstrate that our new SF algorithm is valid and that the use of our $\mathrm{H}_{2}$-regulated $\mathrm{SF}$ prescription does not destroy this agreement. We note that Kravtsov (2003) showed that even a linear local SF law, with a constant SF time scale $t_{\star}$ and a density threshold, can, when spatially averaged on $\lesssim \mathrm{kpc}$ scales, result in a super-linear surface density relation in agreement with the empirical KS law. Matching the observed KS law in cosmological simulations is thus not a good way to distinguish between different SF implementations.

Another cross check with observational data is provided by the demonstration in Fig. 4 that our simulated galaxies exhibit a similar relation between their SFR and total $\mathrm{H}_{2}$ content as the galaxies in the study by Daddi et al. (2010). Our simulated relation has the same slope, and lies in amplitude between what they refer to as the "sequence of starbursts" and the "sequence of disks". Note that a somewhat higher SFR at a given $\mathrm{H}_{2}$ mass may be expected for lower metallicity systems, for which the transition to $\mathrm{H}_{2}$ occurs at higher column densities.

\subsection{Low $\Sigma_{\text {gas }} S F$ Threshold}

Both KT07 and KMT09 simulations exhibit a drop off in $\Sigma_{\mathrm{SFR}}$ at $\Sigma_{\text {gas }} \approx 50 \mathrm{M}_{\odot} \mathrm{pc}^{-2}$. This feature is believed 


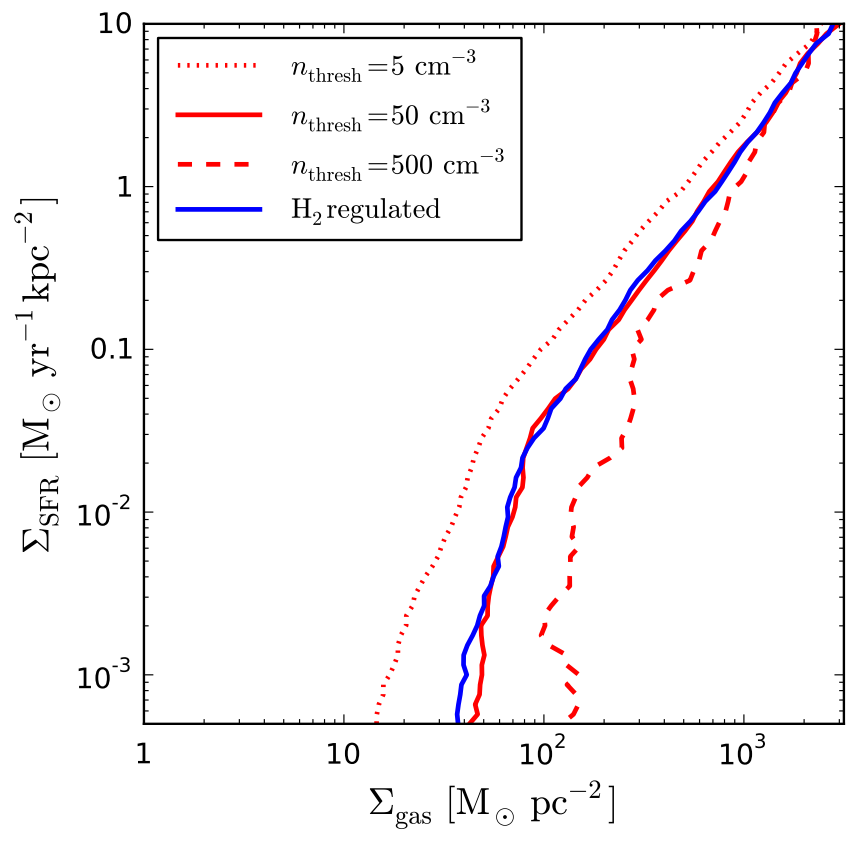

Figure 5. Simulated mean Kennicutt-Schmidt relations for standard SF with three different density thresholds, $n_{\text {thresh }}=$ $5,50,500 \mathrm{~cm}^{-3}$, and the $\mathrm{H}_{2}$-regulated SF. The KT07 and KMT09 lines are from the $z=4$ output, the KT07_low and KT07_high ones from $z=6$.

to correspond to saturation in the atomic hydrogen fraction, with gas becoming primarily molecular at higher surface densities (Bigiel et al. 2008). Since $\Sigma_{\mathrm{SFR}}$ appears to be almost independent of $\Sigma_{\mathrm{HI}}$ and instead correlates primarily with $\Sigma_{\mathrm{H}_{2}}$, this HI saturation point is reflected in a kink towards lower SFR in the KS relation.

In the KT07 simulation, which doesn't account for the atomic to molecular hydrogen transition, this cutoff is reproduced by the density threshold imposed on the SF. In Figure 5 we show how the simulated KS relation depends on the value of this threshold. As expected the relation extends to lower $\Sigma_{\text {gas }}$ for the lower threshold $n_{\text {thresh }}=5 \mathrm{~cm}^{-3}$ case and steepens at a larger value of $\Sigma_{\text {gas }} \approx 100 \mathrm{M}_{\odot} \mathrm{pc}^{-2}$ when the threshold is higher, $n_{\text {thresh }}=500 \mathrm{~cm}^{-3}$ (see also Colín et al. 2010). Once the $\mathrm{SF}$ is regulated by $\mathrm{H}_{2}$, however, this turnover arises naturally: although the curve is noisier due to the smaller statistics, the relation appears to naturally steepen at $\Sigma_{\text {gas }} \approx 50 \mathrm{M}_{\odot} \mathrm{pc}^{-2}$. This confirms the earlier results found by GK10 for a set of cosmological zoom-in galaxy formation simulations including non-equilibrium $\mathrm{H}_{2}$ formation and radiative transfer.

Note that in the KMT09 KS relation (right panel of Fig. 3) there are a few points with very low $\Sigma_{\text {gas }}$ yet non-zero $\Sigma_{\mathrm{SFR}}$, and a roughly corresponding number of points with high $\Sigma_{\text {gas }}$ yet values of $\Sigma_{\text {SFR }}$ significantly below the KS relation. These points arise from star particles having wandered out of the high density cells in which they were born into a neighboring pixel with much lower $\Sigma_{\text {gas }}$. The degree to which this wandering causes a smearing in the KS relation depends on the spatial and temporal averaging scales employed in calculating $\Sigma_{\text {gas }}$ and $\Sigma_{\mathrm{SFR}}$, and on the stars' velocity dispersion.

In local normal disk galaxies stellar velocity dispersions are typically only $5-10 \mathrm{~km} \mathrm{~s}^{-1}$, preventing stars from

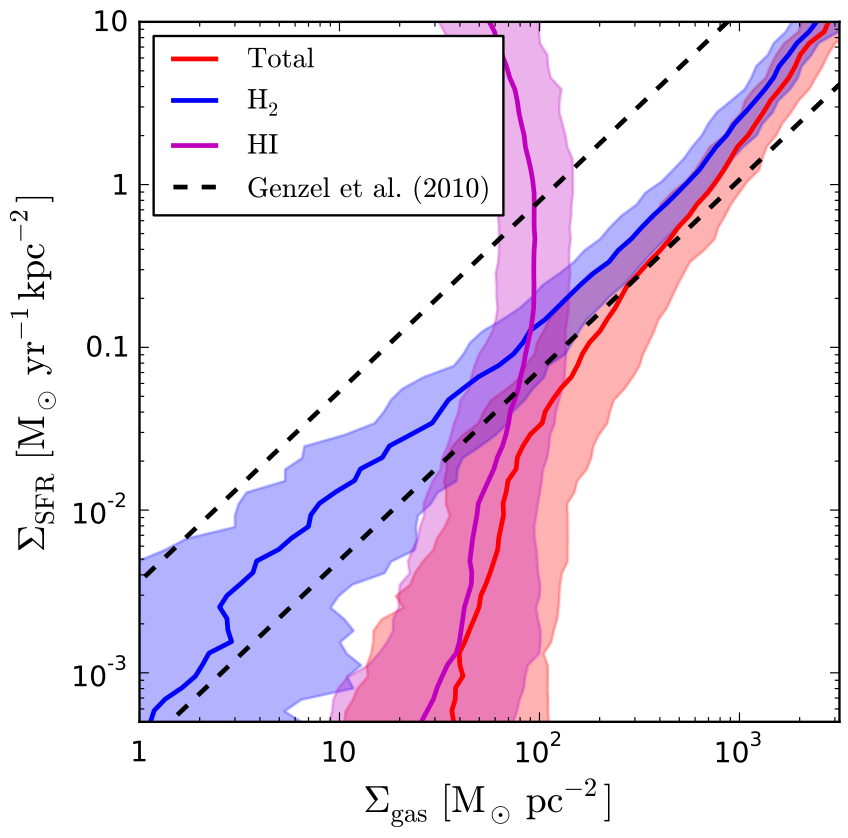

Figure 6. Kennicutt-Schmidt relation for total (red), $\mathrm{H}_{2}$ (blue), and HI (magenta) surface density from the KMT09 simulation at $z=4$. The solid lines indicate the mean relations from the simulations, the shaded regions cover the central $68 \%$ scatter $\left(16^{\text {th }}-84^{\text {th }}\right.$ percentile), and the two dashed lines are the best-fit $\Sigma_{\mathrm{H}_{2}}-\Sigma_{\mathrm{SFR}}$ relations reported by Genzel et al. (2010) for their $z=0-3.5$ samples of "normal" SF galaxies (lower line) and luminous mergers (LIRGs/ULIRGs and SMGs) (upper line).

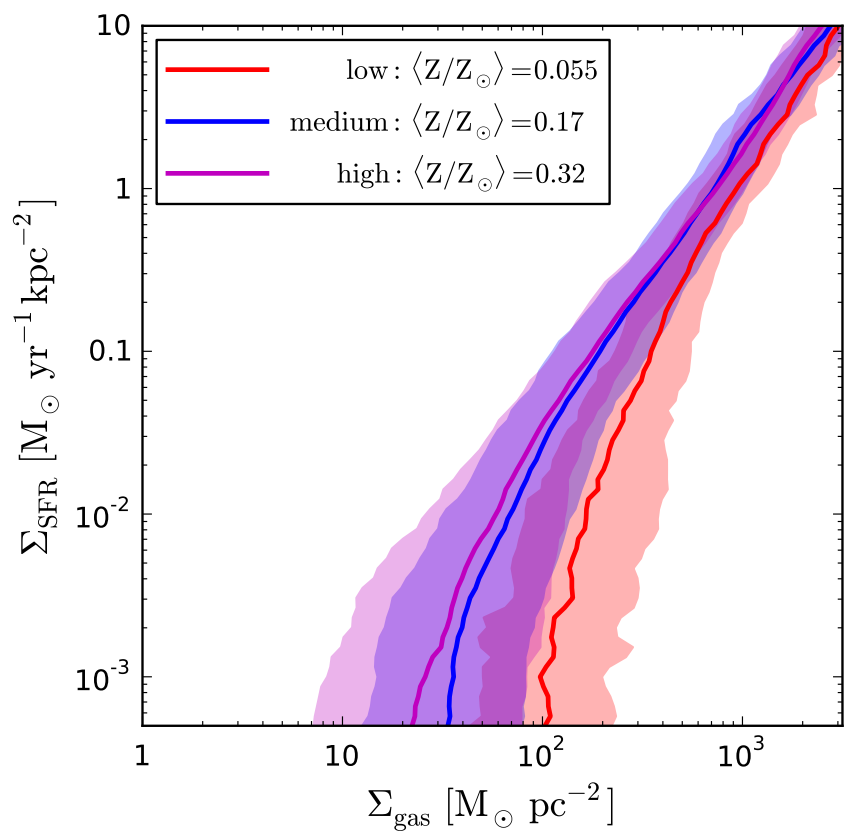

Figure 7. The total gas KS relation for simulated galaxy subsamples split by their mean gas-phase metallicity, from the KMT09 simulation at $z=4$. Solid lines indicate the mean relations from the simulations, and the shaded regions cover the central $68 \%$ scatter $\left(16^{\text {th }}-84^{\text {th }}\right.$ percentile). The lower the metallicity, the higher the $\Sigma_{\text {gas }}$ that is needed for the transition to fully molecular gas, which corresponds to the turn over in the KS relation. 


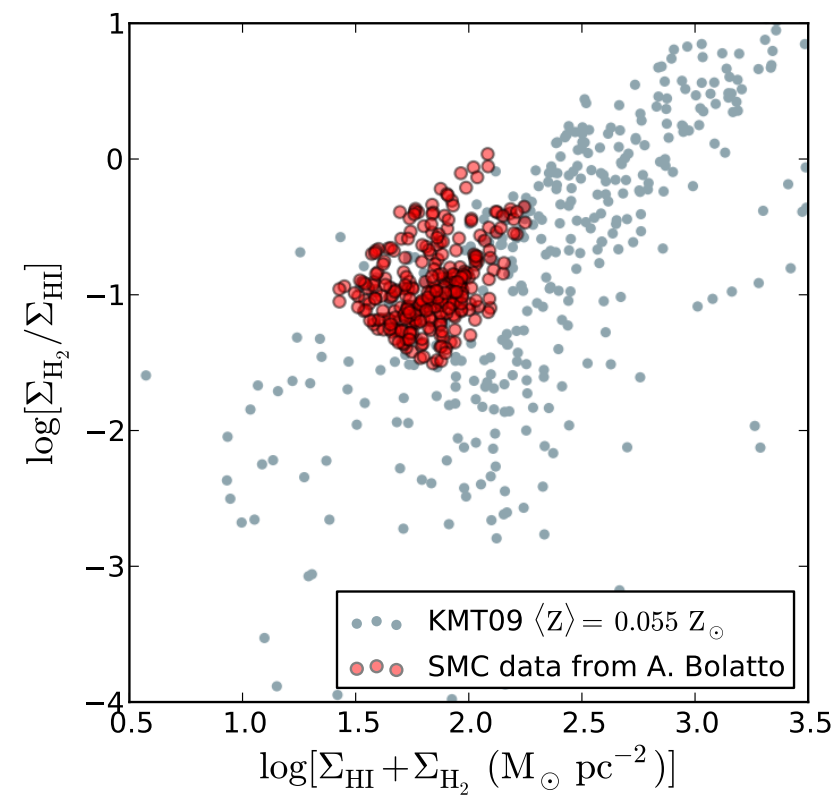

Figure 8. Comparison of the $\mathrm{H}_{2}$-to-HI ratio versus total neutral hydrogen column density $\left(\Sigma_{\mathrm{HI}}+\Sigma_{\mathrm{H}_{2}}\right)$ between SMC data smoothed at $200 \mathrm{pc}$ (from Bolatto et al. 2011) and low metallicity gas in the KMT09 simulation at $z=4$ also smoothed at $200 \mathrm{pc}$. The overlap between simulated and observational data indicates that our model does an adequate job of capturing the atomic-tomolecular transition in real dwarf galaxies.

wandering over more than a small fraction of the $\sim \mathrm{kpc}$ smoothing scale in the $\sim 10$ Myr over which they produce significant ionizing luminosities that may be detected in $\mathrm{H} \alpha$ or other nebular emission lines. Typical velocity dispersions, however, may well be larger at higher redshift (see Cresci et al. (2009) for some empirical evidence for this), as expected if they are set by cosmological accretion (Krumholz \& Burkert 2010) instead of cold disk dynamics. In fact, we find that averaged on 1 kpc scales our galaxies have a mean $1 \mathrm{D}$ stellar velocity dispersion ${ }^{2}$ of $44 \mathrm{~km} \mathrm{~s}^{-1}$ at $\mathrm{z}=4$. In $5 \%$ of all cells (19 in total) the 1D stellar velocity dispersion exceeds $100 \mathrm{~km} \mathrm{~s}^{-1}$. At these velocities wandering becomes more important, and as a result an increased scatter in the KS relation should be expected at high redshift. We have verified that the amount of scatter at low $\Sigma_{\text {gas }}$ increases when we reduce the spatial averaging scale of the surface density maps (see Fig. 90 or increase the temporal SF timescale $t_{\star}($ Feldmann \& Gnedin 2011, see also).

In Figure 6 we compare the $\mathrm{KS}$ relations for the total, atomic, and molecular gas in the KMT09 simulation. The $\mathrm{H}_{2}$ relation does not exhibit a cutoff at low column densities, since we have not imposed any explicit density threshold in the $\mathrm{H}_{2}$-regulated $\mathrm{SF}$ prescription. The $\mathrm{H}_{2}$ relation is shallower than the total gas one, and its slope is in excellent agreement with the observational determination of the slope of the molecular gas KS relation by Genzel et al. (2010). These authors studied a population of "normal" star forming galaxies at $z=0-3.5$ and a population of luminous $z \sim 0$ and $z \sim 1-3.5$ mergers

2 We only include cells containing more than 10 star particles in the average.
(LIRGs/ULIRGs and SMGs) and found that both had equal $\mathrm{H}_{2} \mathrm{KS}$ slopes of 1.17 with about a 1 dex higher normalization for the luminous merger sample. The two best-fit relations from their work are shown as dashed lines in Figure 6 and our $\mathrm{H}_{2} \mathrm{KS}$ relation lies right in between the two. The HI relation instead is much steeper and doesn't extend much beyond $70 \mathrm{M}_{\odot} \mathrm{pc}^{-2}$. The near constant offset at low $\Sigma_{\text {gas }}$ between the total gas and $\mathrm{HI} \mathrm{KS}$ relations is due to the presence of a signification amount of ionized gas on $\sim \mathrm{kpc}$ scales. The overall picture matches the results reported by GK10 and is in qualitative agreement with the empirical findings reported by Bigiel et al. (2008), confirming that the origin of the turn over in the total gas KS relation is indeed the transition from predominantly atomic to fully molecular gas.

\subsection{Metallicity Dependence}

The KS cutoff occurs at somewhat higher $\Sigma_{\text {gas }}$ in both KT07 and KMT than in the observational data from Bigiel et al. (2008). This can be understood as a result of the lower metallicities in our simulated galaxies. We split our 35 galaxy sample into three subsamples of different mean gas phase metallicities, $\left\langle\mathrm{Z} / \mathrm{Z}_{\odot}\right\rangle=0.055,0.17$, and 0.32. Each subsample contains at least 10 galaxies. The highest metallicity galaxy has $\mathrm{Z}=0.56 \mathrm{Z}_{\odot}$, considerably below the closer to solar metallicity sample of $z=0$ field galaxies analyzed by Bigiel et al. (2008). The lower the metallicity of the gas, the higher the total gas surface density that is required in order to provide enough LW shielding to allow the transition to fully molecular gas. The $\Sigma_{\text {gas }}$ scale at which the KS cutoff occurs is thus expected to scale inversely with metallicity, and this is exactly what Figure 7 shows. The cutoff in the KS relation shifts to progressively higher $\Sigma_{\text {gas }}$ for decreasing mean metallicity, occuring at $\gtrsim 100 \mathrm{M}_{\odot} \mathrm{pc}^{-2}$ for the lowest metallicity case. A similar trend was previously reported by GK10. Recent observations of star formation in the Small Magellanic Cloud (SMC) show that the break in the total gas star formation law is indeed shifted to higher surface density by a factor of $\mathrm{Z}_{\odot} / \mathrm{Z}_{\mathrm{SMC}} \sim 5$, in precisely the manner that our models predict (Bolatto et al. 2011). The higher $\Sigma_{\text {gas }}$ cutoff in the KS relation for low metallicity systems may also be responsible for the observational results that $\Sigma_{\mathrm{SFR}}$ in damped Lyman- $\alpha$ (DLA) systems (Wolfe \& Chen 2006) and Lyman-break galaxies (Rafelski et al. 2009, 2011) at $z \approx 3$ appears to lie well below the $z=0 \mathrm{KS}$ relation (see GK10 for a more in depth exploration of this possibility).

The success of our model at capturing the physics governing the gas phase structure in dwarf galaxies is further demonstrated by Fig. 8. There we compare the $\mathrm{H}_{2}$-fraction $\left(\Sigma_{\mathrm{H}_{2}} / \Sigma_{\mathrm{HI}}\right)$ as a function of total neutral hydrogen gas column $\left(\Sigma_{\mathrm{HI}}+\Sigma_{\mathrm{H}_{2}}\right)$ for the low metallicity galaxy sample $\left(\langle\mathrm{Z}\rangle=0.055 \mathrm{Z}_{\odot}\right)$ with observational data for the SMC that has recently become available. Bolatto et al. (2011) have determined a $\mathrm{H}_{2}$ column density map of the SMC at $\sim 12 \mathrm{pc}$ resolution by combining Spitzer IR measurements and radio (ATCA and Parkers) HI data. In Fig. 8 we compared their data smoothed on $\sim 200$ pc scale (kindly provided by A. Bolatto) to our $z=4$ KMT09 simulation data smoothed at the same scale. There is good overlap between simulation and observational data, but in our simulations the distribution of points extends both to higher total gas columns and to 




Figure 9. Dependence of the total gas (left) and $\mathrm{H}_{2}$ (right) KS relation on the smoothing scale $\Delta$ in the KMT09 simulation at $z=4$. The solid lines show the mean relations and the shaded regions cover the central $68 \%$ scatter (for clarity we only show the scatter for $\Delta=$ 10 and $1000 \mathrm{pc}$ ). The mean relation does not show much dependence on averaging scale, but the scatter increases towards smaller $\Delta$, in agreement with observations (Schruba et al. 2010, Liu et al. 2011).

lower $\mathrm{H}_{2}$-fractions than probed by Bolatto et al. (2011).

\subsection{Smoothing Scale Dependence}

In Figure 9 we show the dependence of the total gas and $\mathrm{H}_{2} \mathrm{KS}$ relations on the spatial scale over which the data is smoothed. In addition to our fiducial scale of $200 \mathrm{pc}$, we present the relations for up to twenty times finer and five times coarser smoothing. This range of smoothing scales roughly mimics the variations in the angular resolution (i.e. beam size) of the radio observations used to establish the observational KS relation, ranging from spatially resolved measurements of nearby spiral galaxies with sub-kpc resolution (Kennicutt et al. 2007; Bigiel et al. 2008) to high redshift observations in which a large fraction of the galaxy is contained in a single beam (Kennicutt 1998). We do not see much evidence for a smooth scale dependence, in either total gas or $\mathrm{H}_{2}$ $\mathrm{KS}$ relations. The scatter in the relations, however, increases for smaller smoothing scales, in agreement with observations (Schruba et al. 2010, Liu et al. 2011).

\subsection{Resolution Dependence}

With only seven levels of adaptive mesh refinement, our simulations are unable to resolve the true Jeans length of the cold, molecular gas in star forming galaxies. As discussed in $\S 2$, we resort to an artificial minimum pressure support in order to stabilize gas cells at the highest refinement level against artificial fragmentation. This has the undesirable consequence of making the results of our simulations somewhat dependent on resolution, since additional levels of refinement will allow gas to collapse further and reach higher densities, until the resolution becomes adequate to resolve the true Jeans length. Unfortunately, additional refinement levels come at a computational cost. To run the KMT09_L8 simulation, a clone of KMT09 with one additional refinement level $\left(l_{\max }=8\right)$, down to $z=6$ took about 3 times as long as the KMT09 run. Note that virtually every $l=7$ KMT09 grid was at least partially further refined in KMT09_L8.

In the left panel of Figure 10 we show a comparison of the distribution functions of number density in the maximally refined grid cells in the KMT09 and KMT09_L8 simulations at $z=6$. The additional refinement level has allowed gas to collapse to higher densities. The mean density at $l=7$ is $427 \mathrm{~cm}^{-3}$ in the KMT09 simulation, but $1420 \mathrm{~cm}^{-3}$ at $l=8$ in KMT09_L8. Higher densities will lead to larger a SFR and an increase in $\Sigma_{\text {SFR }} . \Sigma_{\text {gas }}$ smoothed on $\sim$ kpc scales, however, will be unaffected, since it depends only on the total mass enclosed in a given kpc scale column, not the local density. It is not surprising, then, that the simulated KS relation (right panel of Fig. 10 shows that the KMT09_L8 KS relation has somewhat higher amplitude than in KMT09.

It is important to note that, despite its higher resolution, model KMT09_L8 is not necessarily more realistic than KMT09, because it lacks the physics needed to properly model molecular clouds at the increased resolution. In the absence of feedback mechanisms other than supernovae, increasing resolution allows the gas to collapse to ever-higher density, so that the bulk of the molecular gas will always reside near the resolution limit. However, this behavior is not realistic. In observed nearby galaxies, the bulk of the molecular clouds exist at densities of a few hundred $\mathrm{cm}^{-3}$ rather than a few thousand $\mathrm{cm}^{-3}$ (i.e. closer to the mean in KMT09 than KMT09_L8; Blitz 1993), and molecular cloud properties are independent of galactic environment, strongly suggesting internal regulation (Bolatto et al. 2008). Possible mechanisms to provide this regulation include ionized gas pressure (Matzner 2002; Krumholz et al. 2006, Goldbaum et al. 2011), protostellar winds (Nakamura \& Li 2007; Wang et al. 2010), and radiation pressure (Krumholz \& Matzner 2009; Murray et al. 2010; Fall et al. 2010, Hopkins et al. 2011), none of which are included in our simulations. Thus we regard the KS law 

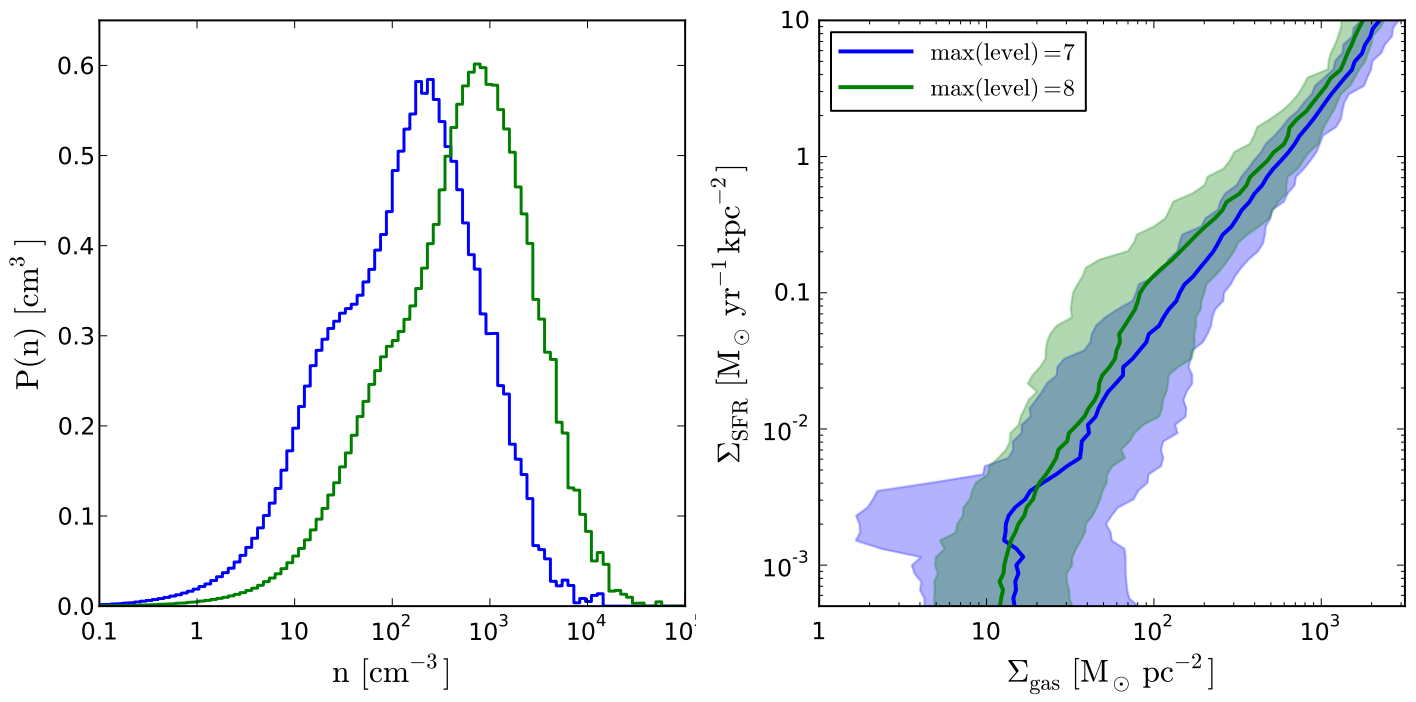

Figure 10. Left: Probability distribution functions of the proper gas number density in the KMT09 and KMT09_L8 simulations at $z=6$ for cells at the maximum refinement level $\left(l_{\max }=7\right.$ and 8 , respectively). Right: The corresponding total gas KS relation. The additional refinement level allows gas in KMT09_L8 to reach higher densities, which in turn results in greater SFR densities, and an increase in the normalization of the KS relation.

we obtain from KMT09 as at least as reliable as the one we obtain from KMT09_L8. Moreover, this comparison yields an important caution: increasing resolution without a corresponding increase in physics does not necessarily yield a better result.

\section{STELLAR MASS FRACTION}

We now turn to the effects of an $\mathrm{H}_{2}$-regulated SF prescription on the stellar content of the dark matter halos in our simulations. In Figure 11 we show plots of the stellar mass fraction $f_{\star}=M_{\star} / M_{h}$ against the total halo mass $\mathrm{M}_{\mathrm{h}}=\mathrm{M}_{\mathrm{DM}}+\mathrm{M}_{\mathrm{gas}}+\mathrm{M}_{\star}$ for simulations with a standard (KT07) and $\mathrm{H}_{2}$-regulated (KMT09_ZFz10 ${ }^{3}$ ) SF prescription. In the normal SF case (left panel), halos with total masses as low as $10^{9} \mathrm{M}_{\odot}$ have been able to form a substantial stellar content, with values of $f_{\star}$ not dropping much below $1 \%$. This is problematic in view of the observational dearth of such dwarf galaxies in the local universe. Given the high volume density of $\mathrm{M}>10^{9} \mathrm{M}_{\odot}$ dark matter halos predicted by $\Lambda \mathrm{CDM}$ structure formation, such a high star formation efficiency would vastly overproduce the faint end of the field dwarf galaxy luminosity function and the abundance of faint Local Group dwarf galaxies.

In Via Lactea II, a collisionless simulation of the formation of a Milky Way's dark matter halo (Diemand et al. 2008), for example, there are $\sim 100$ halos with an identifiable $z=0$ remnant within the host halo's virial volume that had a mass exceeding $10^{9} \mathrm{M}_{\odot}$ at some point in their evolution. Yet of the Milky Way dwarf satellite galaxies, probably only the two Magellanic Clouds, Fornax, and Leo I (and possibly the progenitor of the Sagittarius dwarf) have a stellar mass greater than $10^{7} \mathrm{M}_{\odot}$. The vast majority of such dark matter halos must thus have SF efficiencies well below 1\% (Rashkov et al. 2012). The same is true of faint field galaxies, not just satellites. Com-

\footnotetext{
3 We now focus on KMT09_ZFz10, because the slightly earlier metallicity floor $(z=10$, instead of $z=9$ in KMT09) results in a more gradual suppression of $\left\langle f_{\star}\right\rangle$, see $\S 4.3$
}

parisons of the local universe galaxy luminosity function from SDSS with the predicted DM field halo mass function show that at $z<1$ the stellar mass fraction must have a maximum of $\sim 0.01-0.03$ around $10^{12} \mathrm{M}_{\odot}$, decreasing sharply towards lower masses (Zheng et al.|2007; Conroy \& Wechsler 2009, Guo et al. 2010; Moster et al. 2010 Behroozi et al. 2010).

The most commonly invoked mechanisms to lower the stellar mass fraction in these low mass galaxies are (i) the prevention of gas cooling due to the meta-galactic UV background (Efstathiou 1992: Kauffmann et al.1993; Bullock et al. 2000) and (ii) stellar feedback from massive winds and supernovae (Larson 1974, Kauffmann et al. 1993 Benson et al. 2002). Our simulations include a model for the UV background, via a spatially uniform, optically thin radiation field that accounts for the UV emissivity from both quasars and galaxies ${ }^{4}$ (Haardt \& Madau 2001). By itself this feedback does not appear to be able to suppress star formation in $10^{9}-10^{10} \mathrm{M}_{\odot}$ halos at $z>4$ in our simulations. As discussed in $\S 2.3$, we do include a supernova feedback prescription in our simulation, but in a form known to be insufficiently strong to effect the necessary reduction in SF efficiency or to reproduce observed galactic outflows. This is exemplified by the high stellar mass fractions $\left(f_{\star} \approx 0.06\right.$, a factor $5-10$ higher than what is allowed by observational constraints at much lower redshift, $z \lesssim 1$ ) even in our most massive halos $\left(10^{10}-10^{11} \mathrm{M}_{\odot}\right)$ (see also Avila-Reese et al. 2011).

It's reasonable to expect a similar, or even greater, suppression of $f_{\star}$ for lower mass halos, whose shallower potential wells should make it easier for winds and supernovae to expel their gas. However, Font et al. (2011) find that in order to simultaneously match the luminosity function of Local Group dwarf galaxies and their observed mass-metallicity relation, the strength of stellar feedback must saturate in halos with $V_{\max } \lesssim 65 \mathrm{~km} \mathrm{~s}^{-1}$ $\left(\approx 5 \times 10^{10} \mathrm{M}_{\odot}\right)$. Furthermore, a physically realistic

4 The galactic contribution dominates the UV background at this early epoch. 


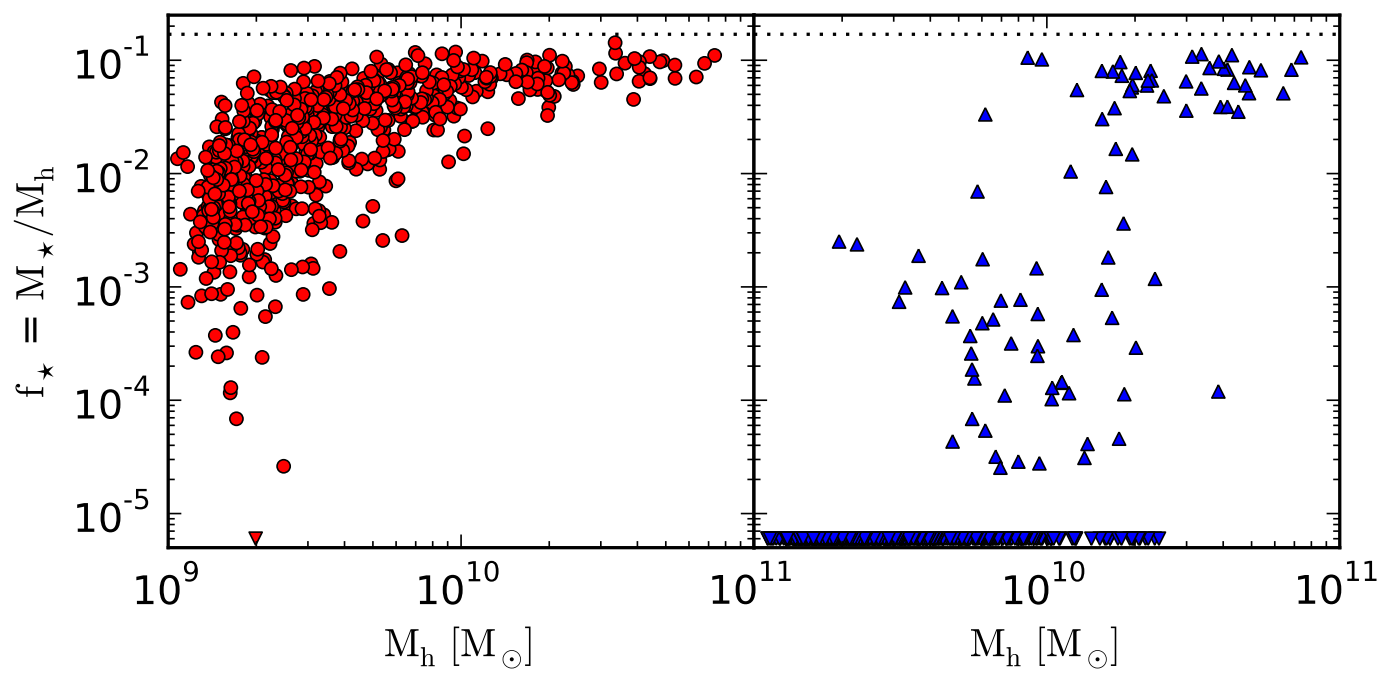

Figure 11. Stellar mass fraction $\mathrm{f}_{\star}$ vs. total halo mass in a simulation with standard SF (KT07, left) and with $\mathrm{H}_{2}$-regulated $\mathrm{SF}$ (KMT09_ZFz10, right) at $z=4$. The dotted horizontal line indicates an $\mathrm{f}_{\star}$ equal to the cosmic baryon fraction $\Omega_{\mathrm{b}} / \Omega_{\mathrm{M}}$, i.e. a $100 \%$ gas to star conversion efficiency.

modeling of stellar feedback processes in cosmological numerical simulations is beyond current computational capabilities, and it remains unclear how well the various sub-grid physics implementations discussed in the literature actually capture the true nature of this feedback. Although we don't doubt that stellar feedback processes play an important role in regulating star formation in low mass halos, we now show that regulating SF by the $\mathrm{H}_{2}$ abundance may play an equally important role in lowering stellar mass fractions in low mass $\left(<10^{10} \mathrm{M}_{\odot}\right)$ halos, as previously suggested by G09, GK10.

The right panel of Figure 11 shows that our $\mathrm{H}_{2}$ regulated SF prescription introduces a threshold below which the stellar mass content of halos is strongly suppressed. At halo masses greater than $10^{10} \mathrm{M}_{\odot}$, the star formation efficiency is only mildly reduced, dropping from $\left\langle f_{\star}\right\rangle=0.062$ to 0.035 . Between $5 \times 10^{9} \mathrm{M}_{\odot}$ and $10^{10} \mathrm{M}_{\odot}$, only a small fraction of halos (about $10 \%$ ) has been able to form stars and only with reduced SF efficiency, and at even lower halo masses star formation has been almost completely suppressed. We visually demonstrate this suppression in Figure 12 , where we show direct comparisons of the baryonic structure $\left(\Sigma_{*}, \Sigma_{\text {gas }}, \Sigma_{\mathrm{H}_{2}}\right.$, and density-weighted metallicity) between the KT07 and KMT09_ZFz10 simulations for two representative halos; one high mass halo $\left(\mathrm{M}_{\mathrm{h}}=2.0 \times 10^{11} \mathrm{M}_{\odot}\right)$ in which $\mathrm{H}_{2^{-}}$ regulation has not had a big effect, and one low mass halo $\left(\mathrm{M}_{\mathrm{h}}=5.8 \times 10^{9} \mathrm{M}_{\odot}\right)$, in which the stellar mass fraction has been suppressed by nearly one order of magnitude from $\mathrm{f}_{\star}=0.057$ to 0.0069 . Many more halos like these exist in our simulations.

Figure 13 shows the baryonic content of halos in KT07 and KMT09_ZFz10 as a function of their mass. We plot mean mass fractions in $\mathrm{M}_{\mathrm{h}}$-bins of width 0.25 dex of the total baryonic content, stars, neutral gas $(\mathrm{HI}+\mathrm{HeI}+$ $\mathrm{H}_{2}$ ), ionized gas ( $\left.\mathrm{HII}+\mathrm{HeII}+\mathrm{HeIII}\right), \mathrm{H}_{2}$, and metals. The total baryonic mass fraction remains equal to the cosmic mean down to $\sim 2 \times 10^{9} \mathrm{M}_{\odot}$. The slight drop in the total baryonic and neutral gas fractions at lower halo masses can be attributed to the meta-galactic UV back- ground, which is able to ionize and heat most of the gas, preventing it from falling into the halo. The mass fractions of $\mathrm{H}_{2}$, metals, and stars roughly trace each other. In KT07, the stellar mass fraction decreases gradually from $\sim 0.4$ of the cosmic baryon fraction at $\mathrm{M}_{\mathrm{h}}=10^{11} \mathrm{M}_{\odot}$ to 0.25 at $10^{10} \mathrm{M}_{\odot}$, before the combined actions of UV background and (weak) supernova feedback further reduce it to $\sim 0.01$ at $10^{9} \mathrm{M}_{\odot}$. In KMT09_ZFz10, on the other hand, the stellar mass fraction is suppressed already at much higher halo masses, dropping below 0.1 of the cosmic mean at $10^{10} \mathrm{M}_{\odot}$ and cutting off completely at $4 \times 10^{9} \mathrm{M}_{\odot}$. Note that because we have included halos with $\mathrm{M}_{\star}=0$ in the calculation of the mean $\mathrm{f}_{\star}$ it should not be viewed as a typical value for any individual halo, but as a population average. With $\mathrm{H}_{2}$-regulation the $\mathrm{SF}$ is quenched in low mass halos without heating and removing much of the gas, contrary to the effects of efficient supernova feedback.

Why should a locally $\mathrm{H}_{2}$-regulated $\mathrm{SF}$ prescription be sensitive to the total halo mass? Recall that with the two-phase equilibrium assumption (KMT09 model), the local $\mathrm{H}_{2}$ abundance is completely determined by the $\mathrm{HI}$ column density and the metallicity of the gas. A reduced SF efficiency in low mass halos could thus be due to either lower column densities or to lower metallicities than in more massive halos, or to a combination of the two.

Figure 14 reveals that lower metallicities are primarily responsible. The figure shows two-dimensional volumetric probability density functions (phase diagrams) of total gas column density $\Sigma_{\text {gas }}$ and metallicity $\mathrm{Z}$, for $l=7$ grid cells in $\mathrm{M}<10^{10} \mathrm{M}_{\odot}$ (left panel) and $\mathrm{M}>10^{10} \mathrm{M}_{\odot}$ halos (right panel) halos. The color scale indicates the volume fraction at a given $\left(\Sigma_{\text {gas }}, \mathrm{Z}\right)$, and the diagonal lines represent contours of constant $f_{\mathrm{H}_{2}}$, according to the KMT09 prescription. The one-dimensional distributions at the top and sides of the figure show that while both low and high mass halos have comparable column density distributions, the metallicity distribution peaks at $\mathrm{Z} / \mathrm{Z}_{\odot} \approx 0.25$ for the high mass halos, but at $\mathrm{Z} / \mathrm{Z}_{\odot}=10^{-3}$ for the low mass halos. Recall that we 


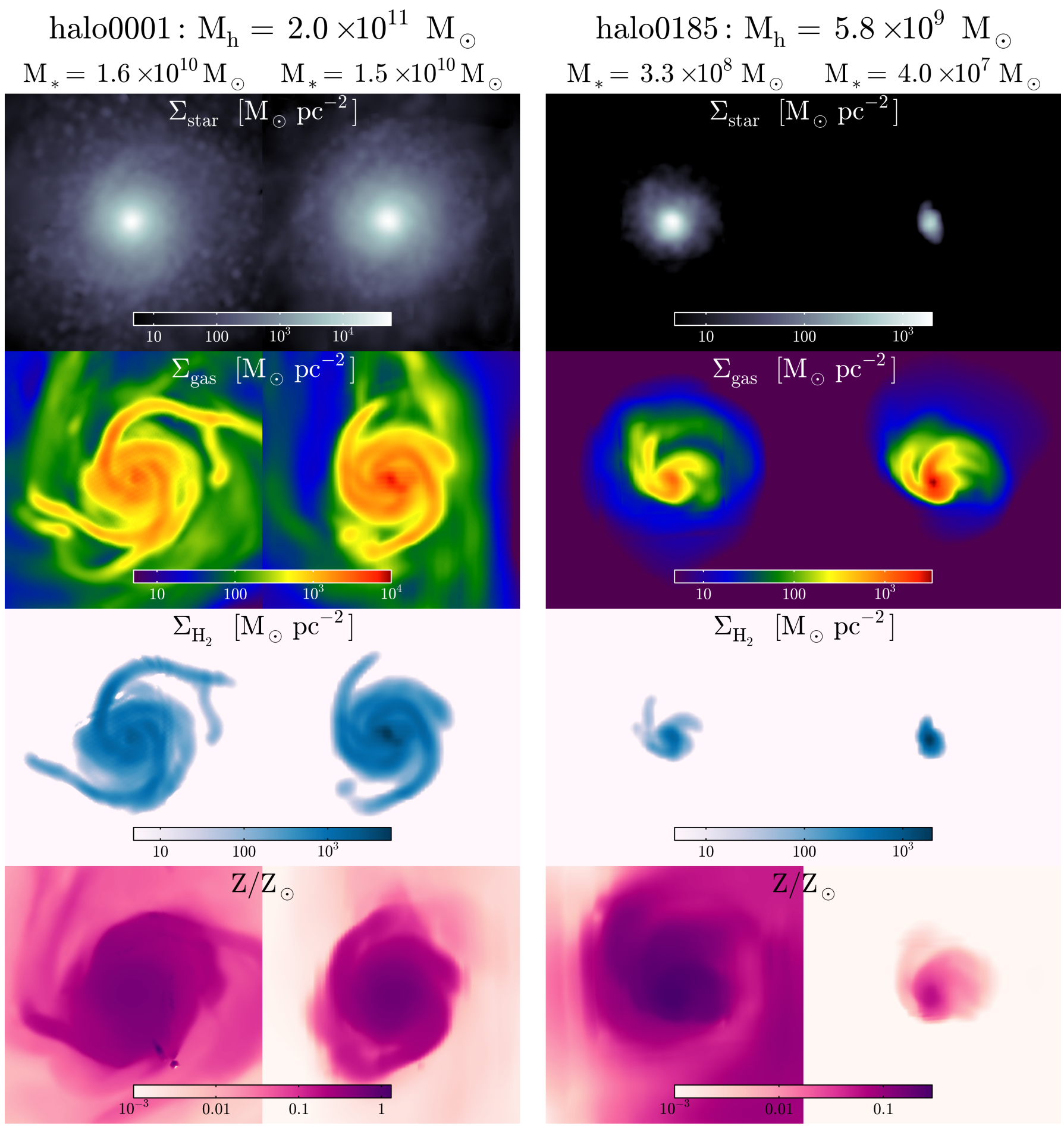

Figure 12. A comparison of the baryonic structure of two identical halos, one with high total mass (halo0001, left panel) and one with low total mass (halo0185, right panel), between the KT07 (left columns) and KMT09_ZFz10 (right columns) simulations at $z=4$. From top to bottom, we show surface densities of stellar mass, total gas, and $\mathrm{H}_{2}$, and a density-weighted projection of metallicity, in a $5 \times 5 \mathrm{kpc}$ region centered on the galaxies. These are merely two representative halos, and many more like them exist in our simulations. The stellar content is greatly suppressed in the KMT09 low mass halo. 


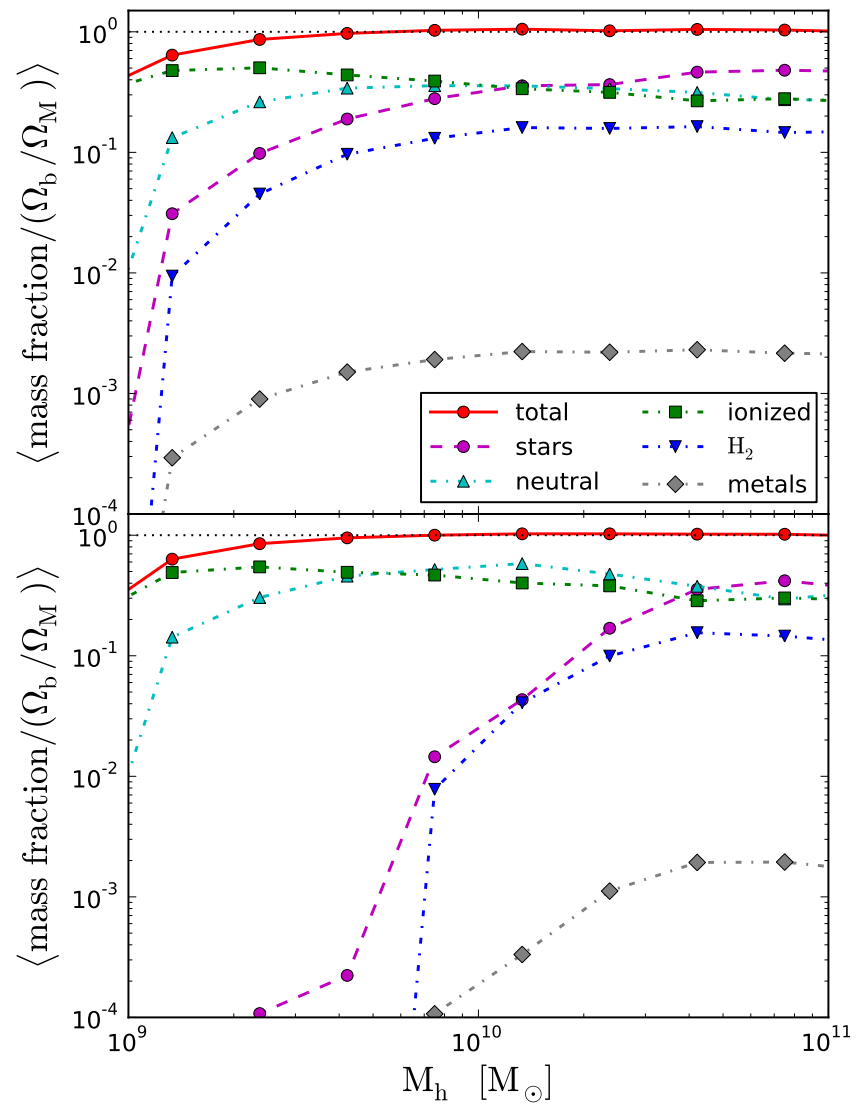

Figure 13. The baryonic content of halos versus their total mass, for the KT07 (top panel) and KMT09_ZFz10 (bottom panel) simulations at $z=4$. We show mean mass fractions normalized to the cosmic baryon fraction $\left(\Omega_{b} / \Omega_{M}\right)$ in $\mathrm{M}_{\mathrm{h}}$-bins of width 0.25 dex for the total baryonic content (stars + all gas + metals; solid lines with circles), stars (dashed with circles), neutral gas (HI $+\mathrm{HeI}+$ $\mathrm{H}_{2}$; dot-dashed with upward triangles), ionized gas (HII $+\mathrm{HeII}+$ HeIII; dot-dashed with squares), $\mathrm{H}_{2}$ (dot-dashed with downward triangles), and metals (dot-dashed with diamonds). Note that although an $\mathrm{H}_{2}$ curve is plotted in the KT07 panel, $\mathrm{H}_{2}$ has no effect in that simulation.

imposed a $\mathrm{Z} / \mathrm{Z}_{\odot}=10^{-3}$ metallicity floor at $z=9$, so this indicates that low mass halos typically remain unenriched. This lack of metals in low mass halos is responsible for a reduced $\mathrm{H}_{2}$ abundance, and hence a suppressed stellar mass fraction.

As we discuss in the remainder of this section, a number of factors, both of physical nature and pertaining to the limitations of our simulations, affect the mass scale and the degree of the $\mathrm{f}_{\star}$ suppression due to $\mathrm{H}_{2}$-regulated SF. A complete suppression of SF in halos with masses below $5 \times 10^{9} \mathrm{M}_{\odot}$ is too strong of an effect to reproduce the luminosity function of Local Group satellites, many of which are consistent with having formed in $\lesssim 10^{9} \mathrm{M}_{\odot}$ halos (Madau et al. 2008). In this work, we demonstrate that $\mathrm{H}_{2}$-regulated $\mathrm{SF}^{\prime}$ can have important consequences for the star formation efficiency in low mass halos, but openly acknowledge that the true nature of this suppression will depend on details of the star formation processes, stellar feedback, and metal enrichment that are not captured with sufficient fidelity in our simulations.

\subsection{Dependence on Two-Phase Equilibrium}

The assumption of two-phase equilibrium between a Cold Neutral Medium (CNM), hosting molecular clouds and SF, and the surrounding Warm Neutral Medium (WNM) is not uncontroversial. As discussed in Krumholz et al. (2009), shocks from supernova explosions or supersonic ISM turbulence, for example, likely temporarily drive the surrounding gas out of pressure balance, in which case the typical gas density could differ significantly from the value estimated from two-phase equilibrium. The time to re-establish equilibrium between a CNM and WNM can be comparable (few Myr, Wolfire et al. (2003)) to the shock recurrence time scale, and hence it is prudent to consider the effects of the KMT09 model without the two-phase equilibrium assumption.

In this case the molecular hydrogen fraction is no longer independent of the $\mathrm{H}_{2}$-dissociating $\mathrm{LW}$ background, and absent a full radiation transfer treatment (G09) we need to externally specify its intensity. For computational ease we have considered here only the case of a constant, spatially uniform LW intensity. Of course it would be preferable to tie the LW intensity to the local $\mathrm{SFR}$, averaged over $\sim \mathrm{kpc}$ scales and tens of Myr, but we defer the investigation of this more realistic treatment to future work.

In Figure 15 we show $\left\langle\mathrm{f}_{\star}\right\rangle$ vs. $\mathrm{M}_{\mathrm{h}}$ at $z=5$ for simulations KMT09_FLW $\{1,10,100,1000\}$, with values of the LW intensity ranging from 1 to 1000 times the $z=0$ Milky Way value of $7.5 \times 10^{-4} \mathrm{LW}$ photons $\mathrm{cm}^{-3}$ (Draine 1978). For clarity we have plotted the mean values of $f_{\star}$ in bins of $\mathrm{M}_{\mathrm{h}}$ of width 0.25 dex. The means include halos with $f_{\star}=0$, which implies that these values of $\left\langle f_{\star}\right\rangle$ should thus be viewed as population averages, rather than representative values for any individual halo. The results from the standard SF (KT07) and the two-phase equilibrium (KMT09) simulations are included for comparison. The lowest LW intensity case (KMT09_FLW1) is almost indistinguishable from the standard SF model. In this case the dissociating flux is not strong enough to affect the $\mathrm{H}_{2}$ abundance even in the lowest mass halos. As the intensity of the LW background is increased, the halo mass at which $\mathrm{H}_{2}$ formation begins to be suppressed increases in proportion. At 1000 times the Milky Way's LW intensity (lowest dotted line), the suppression mass almost reaches the value in the two-phase equilibrium model. Recall that the KMT09_FLW simulations were run with an effective sub-grid clumping factor of 30 $\left(\left(\sigma_{d,-21} / \mathcal{R}_{-16.5}\right)=1 / 30\right)$, and the resulting enhanced $\mathrm{H}_{2}$ recombination rate is partially responsible for the reduced SF suppression compared to the two phase equilibrium simulation. However, while the absolute strength of the SF suppression in the simulations without two-phase equilibrium is sensitive to the value of the clumping factor, the trend with the intensity of the LW background is not.

\subsection{Resolution Dependence}

In Figure 16 we compare $f_{\star}$ vs. halo mass for the KMT09_L8 simulation to the results from KT07 and KMT09. While there still is a suppression of star formation in low mass halos compared to KT07, the scale at which this suppression occurs has shifted to lower masses, around $3 \times 10^{9} \mathrm{M}_{\odot}$. Considering our earlier finding $(\S 3.4$ ) that the additional resolution increases the SF 


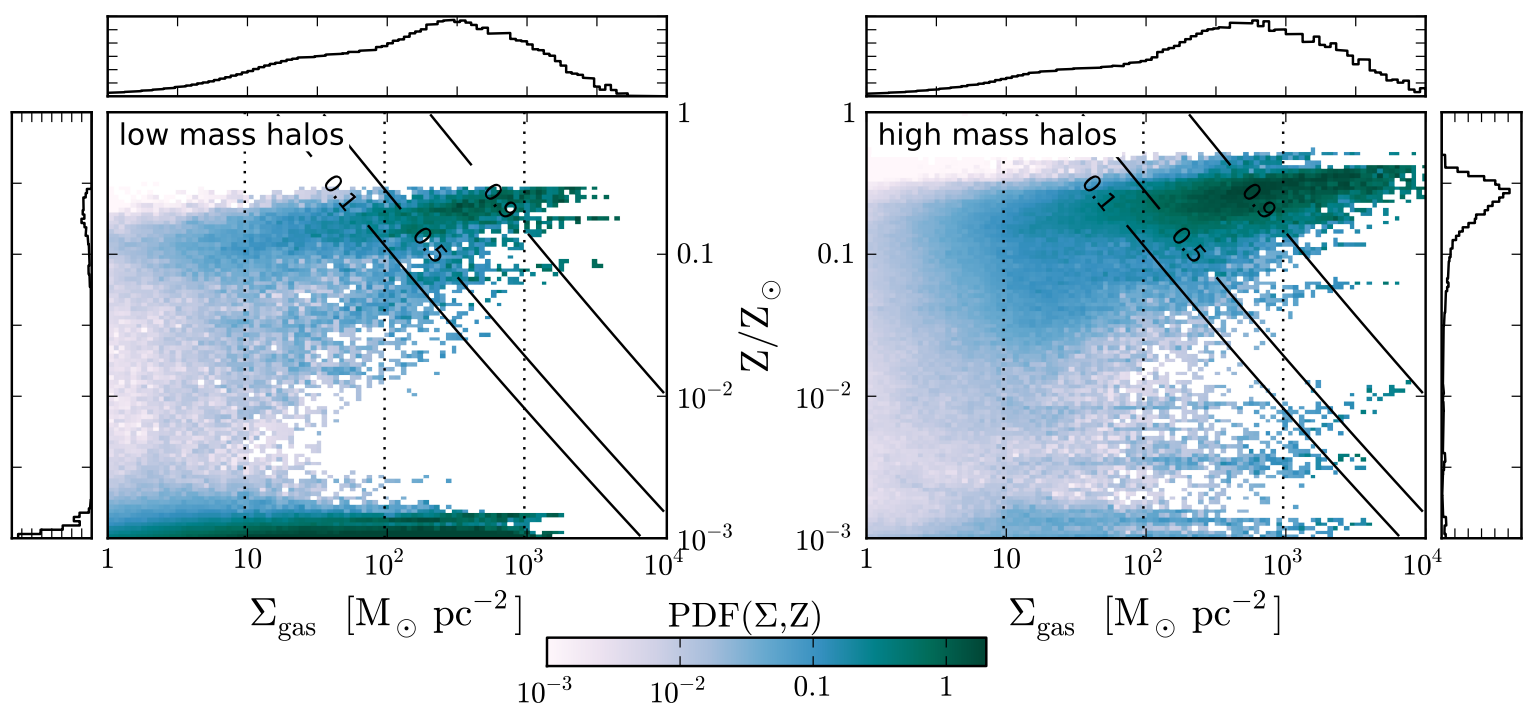

Figure 14. $2 \mathrm{D}$ phase diagrams of $\Sigma_{\text {gas }}$ vs. $\mathrm{Z}$ determined at $l_{\max }=7$ in the KMT09 simulation at $z=5$, for low mass $\left(\mathrm{M}<10^{10} \mathrm{M}_{\odot}\right.$, left panel) and high mass $\left(\mathrm{M}>10^{10} \mathrm{M}_{\odot}\right.$, right panel) halos. Contours of constant $f_{\mathrm{H}_{2}}$ (at 0.1, 0.5, and 0.9) are plotted with solid lines. For reference we show with dotted lines the column densities corresponding to the $3 \mathrm{D}$ density thresholds employed in the KT07 $\left(50 \mathrm{~cm}^{-3}\right)$, KT07_low $\left(5 \mathrm{~cm}^{-3}\right)$, and KT07_high $\left(500 \mathrm{~cm}^{-3}\right)$ simulations. The majority of gas in low mass halos has very little or no $\mathrm{H}_{2}$, and this appears to be primarily due to lower metallicities, not lower column densities.

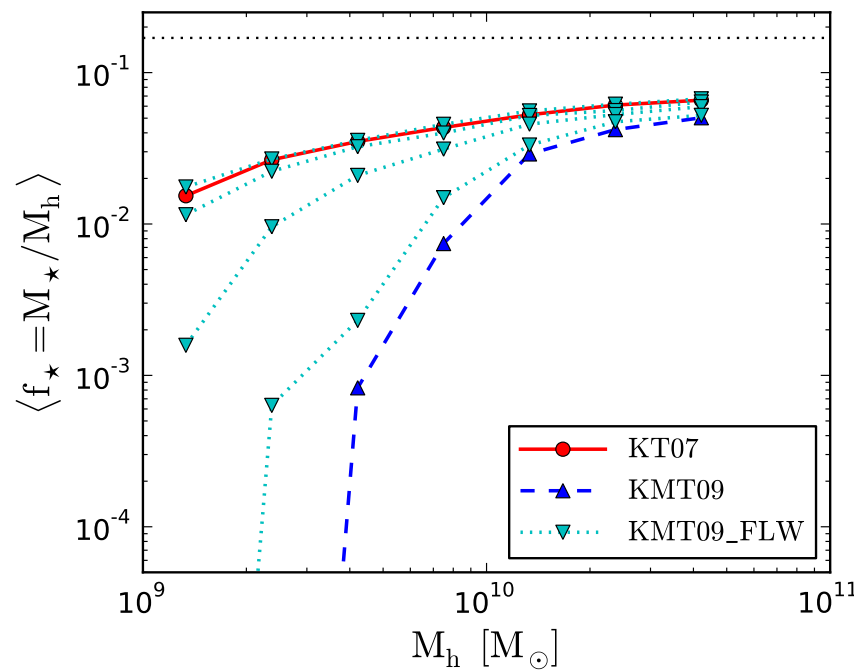

Figure 15. $\left\langle\mathrm{f}_{\star}\right\rangle$ vs. $\mathrm{M}_{\mathrm{h}}$ at $z=5$ for the four KMT09 simulations without the two phase equilibrium assumption and a spatially uniform Lyman-Werner radiation field with intensity equal to 1,10 , $100,1000 \times$ the mean Milky Way value (dotted lines with downward triangles; increasing LW intensity from top to bottom). We plot the mean of $f_{\star}$ in $M_{h}$-bins of width 0.25 dex. The means include halos with $f_{\star}=0$. For comparison we also show the original KT07 (solid line with red circles) and two-phase equilibrium KMT09 results (dashed line with upward triangles). Note that for the KMT09_FLW simulations (but not for KMT09) we apply a "clumping factor" of 30 to the $\mathrm{H}_{2}$ formation rate (see text for details).

rates in KMT09_L8, it is perhaps not surprising that the $\mathrm{f}_{\star}$-suppression mass scale also exhibits some resolution dependence. In this case the sensitivity to the maximum resolution arises because the increase in density afforded by the additional refinement level exceeds the factor of two reduction in grid cell width, thus leading to a net increase in column densities on the finest grid cells. The enhanced LW shielding eases the transition to the molec-

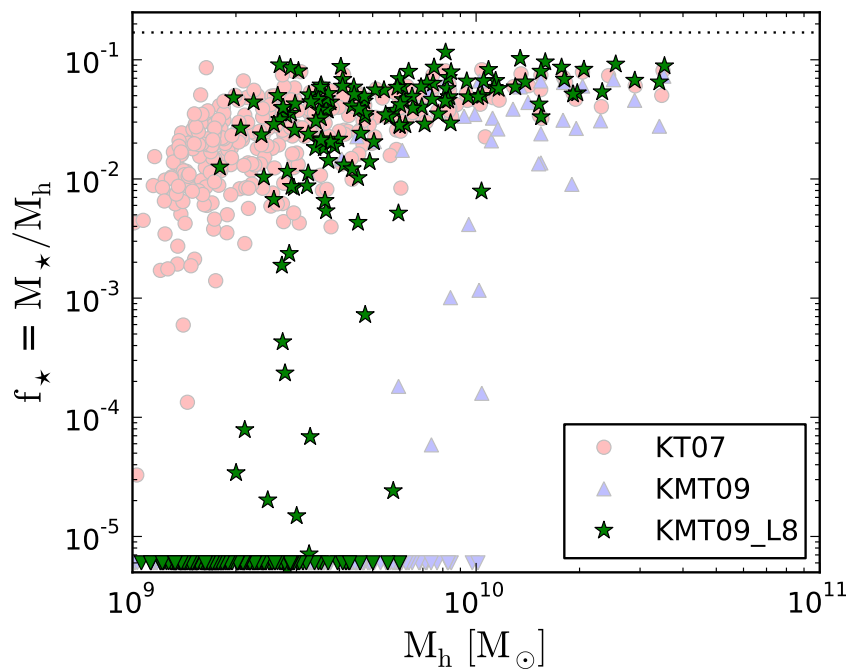

Figure 16. $\mathrm{f}_{\star}$ vs. halo mass (at $z=6$ ) for the KMT09_L8 simulation, which has one additional refinement level compared to KMT09 and KT07. The halo mass scale at which star formation is suppressed due to the inability of gas to become molecular is lower than in the KMT09 simulation. The true value of this suppression mass scale in nature will depend on the strength of stellar feedback, metal mixing, and other processes not resolved in our simulations.

ular phase, and allows star formation to occur in lower mass halos.

The exact value of this transition will depend on the details of the star formation and stellar feedback processes on scales below our current resolution limit, as we discussed in $\S 3.4$. However, our fiducial $l_{\max }=7$ grid resolution of $76.3 \times 5 /(1+z)$ proper parsec is comparable to the observed size of giant atomic-molecular cloud complexes, and our $l=7$ grid cell averaged densities (Fig. 10, left panel) are in good agreement with observational estimates of their average densities (Blitz 1993). We thus believe that the resolution of our fiducial simu- 


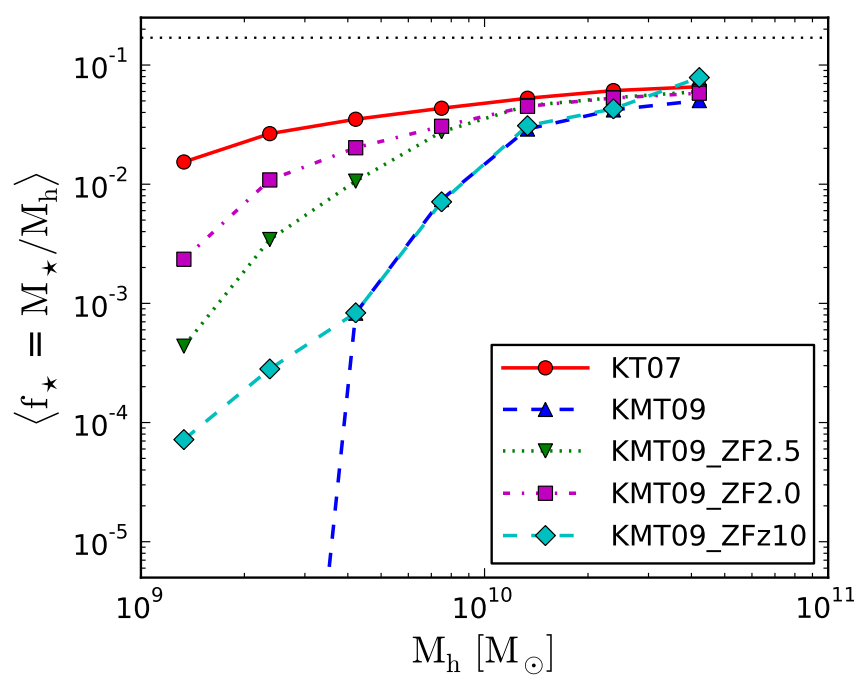

Figure 17. The dependence of $\left\langle\mathrm{f}_{\star}\left(\mathrm{M}_{\mathrm{h}}\right)\right\rangle$ at $z=6$ on the metallicity floor. We plot the mean of $f_{\star}$ in $\mathrm{M}_{\mathrm{h}}$-bins of width 0.25 dex at $z=6$ for KT07 (solid lines with circles) and three versions of KMT09 with increasing values of the $z=9$ metallicity floor, $\left[\mathrm{Z}_{\text {floor }}\right]=-3.0$ (dashed with upward triangles), -2.5 (dotted with downward triangles), and -2.0 (dot-dashed with squares), and one case with $\left[\mathrm{Z}_{\text {floor }}\right]=-3.0$ applied at $z=10$ (dashed with diamonds). The means include halos with $f_{\star}=0$. Note that a simulation with $\log _{10}\left(\mathrm{Z}_{\text {floor }} / \mathrm{Z}_{\odot}\right)=-4.0$ at $z=9$ produced no stars at all.

lations $\left(l_{\max }=7\right)$ is well matched to the problem we are studying.

\subsection{Metallicity Floor Dependence}

In Figure 17 we show how the $f_{\star}$ suppression depends on the amplitude and time of the metallicity floor that we impose early in the simulations to mimic the enrichment from Pop.III supernovae. Higher values of $\left[\mathrm{Z}_{\text {floor }}\right]$ and an earlier time allow SF to occur in lower mass halos. Note that the KMT09_ZF4.0 simulation $\left(\left[\mathrm{Z}_{\text {floor }}\right]=-4.0\right)$ produced no stars at all. Imposing the metallicity floor at $z=10$ instead of $z=9$ has the particularly interesting result of allowing a small amount of star formation in very low mass halos $\left(\mathrm{M}_{\mathrm{h}}<5 \times 10^{9} \mathrm{M}_{\odot}\right)$, which may help to alleviate the tension with the faint-end of the Local Group satellite galaxy luminosity function.

For a given $\mathrm{Z}_{\mathrm{floor}}$, a halo's ability to form stars is determined by the highest column densities that its gas can condense to prior to the enrichment from the first star particles in our simulation. Gas in more massive halos is able to reach higher column densities, owing to their earlier collapse times and deeper potentials. Below some halo mass scale, gas will not be able to reach sufficiently high column densities to turn molecular and allow star formation to occur. The necessary column densities depend on metallicity: at $[\mathrm{Z}] \equiv \log _{10}\left(\mathrm{Z} / \mathrm{Z}_{\odot}\right)=-3.0$, gas must reach a column density of $6.7 \times 10^{3}\left(1.4 \times 10^{4}\right.$ $\left.8.0 \times 10^{4}\right) \mathrm{M}_{\odot} \mathrm{pc}^{-2}$ in order to be $10(50,90)$ per cent molecular. At $[\mathrm{Z}]=-2.5$ the required column is reduced to $2.3 \times 10^{3}\left(4.9 \times 10^{3}, 3.0 \times 10^{4}\right) \mathrm{M}_{\odot} \mathrm{pc}^{-2}$, and it is only $8.0 \times 10^{2}\left(1.7 \times 10^{3}, 1.0 \times 10^{4}\right) \mathrm{M}_{\odot} \mathrm{pc}^{-2}$ at $[\mathrm{Z}]=-2.0$. This naturally explains the observed dependence of the $f_{\star}$ suppression scale on the amplitude of the metallicity floor. It implies that the true $f_{\star}$ suppression realized in nature will depend on the details of the enrichment his-

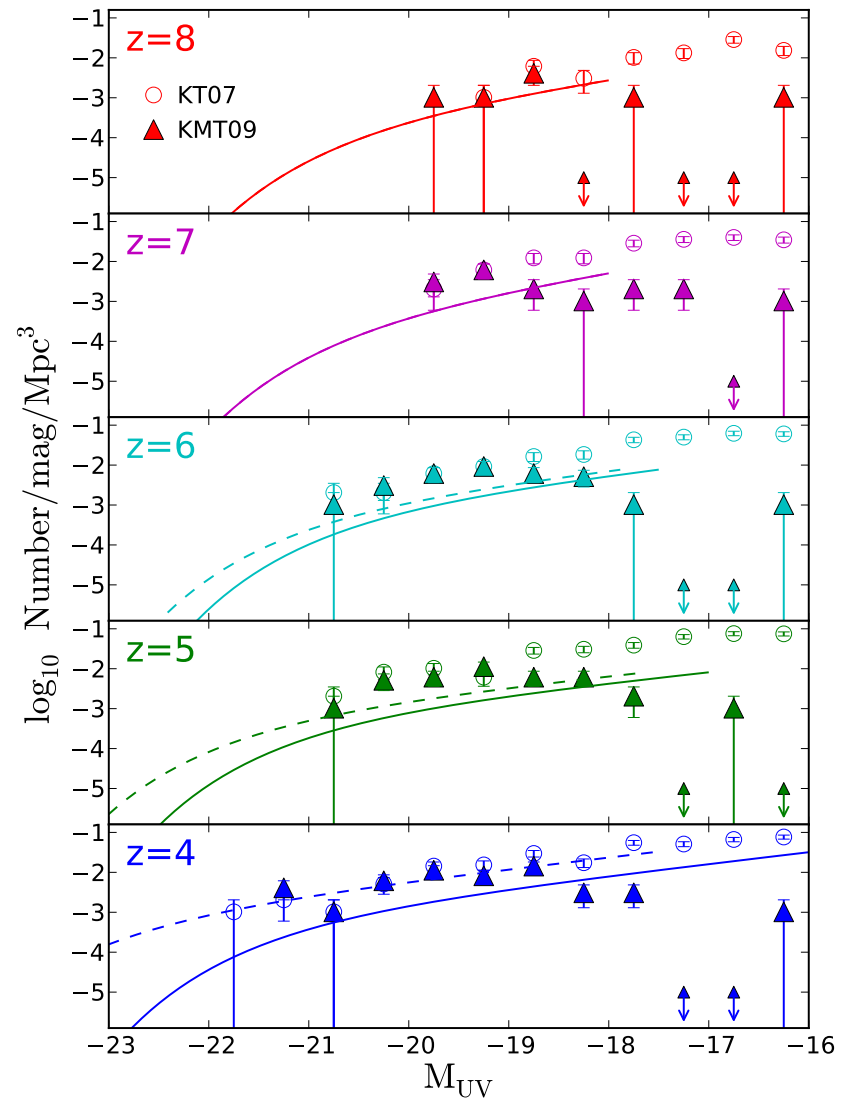

Figure 18. The luminosity function in the KT07 (open circles) and KMT09_ZFz10 (solid triangles) simulations compared to observational results. We calculate UV luminosities from the simulated galaxies' $\mathrm{SFR}$ according to $\mathrm{L}_{\mathrm{UV}}=8.0 \times 10^{27}\left(\mathrm{SFR} / \mathrm{M}_{\odot} \mathrm{yr}^{-1}\right) \mathrm{erg}$ $\mathrm{s}^{-1} \mathrm{~Hz}^{-1}\left(\mathrm{M}_{\mathrm{UV}}=51.63-2.5 \log _{10}\left(\mathrm{~L}_{\mathrm{UV}} / \mathrm{erg} \mathrm{s}^{-1} \mathrm{~Hz}^{-1}\right)\right)$, corresponding to a Salpeter IMF from 0.1 to $125 \mathrm{M}_{\odot}$ (same as Bouwens et al. 2011). Error bars are statistical only. The solid lines are the uncorrected luminosity functions reported by Bouwens et al. (2007) 2011), and the dashed lines the same relations corrected for dust extinction by $(1.55,0.625,0.375,0,0)$ magnitudes at $z=(4,5,6,7,8)$ (cf. Table 8 in Bouwens et al. 2011).

tory of a given halo, which should lead to a broadening of the $M_{h}$ dependence of the $f_{\star}$ suppression.

\section{COMPARISONS WITH HIGH REDSHIFT OBSERVATIONS}

\subsection{Evolution of the Luminosity Function}

In Figure 18 we present a comparison of the luminosity functions (LF) from the KT07 and KMT09_ZFz10 simulations and the recent high redshift determinations from Bouwens et al. (2007, 2011) based on deep HST ACS and WFC3 observations of $B(\langle z\rangle=3.8), V(5.0), i$ (5.9), $z$ (6.8), and $Y$-band (8.0) dropout galaxies. Following Bouwens et al. (2011, hereafter B10), we determine UV luminosities from the simulated SFR according to $\mathrm{L}_{\mathrm{UV}}=8.0 \times 10^{27}\left(\mathrm{SFR} / \mathrm{M}_{\odot} \mathrm{yr}^{-1}\right) \mathrm{erg} \mathrm{s}^{-1} \mathrm{~Hz}^{-1}$, corresponding to a Salpeter IMF from 0.1 to $125 \mathrm{M}_{\odot}$ and a constant SFR over $\gtrsim 100$ Myr (Madau et al. 1998). We calculate SFR from our simulated galaxies by summing the mass of all young star particles with ages less than $\tau_{\star}=30 \mathrm{Myr}$ and dividing by the SF time scale,

$$
\mathrm{SFR}=\sum_{\text {age }<\tau_{\star}} \frac{m_{\star}}{\tau_{\star}} .
$$


The assumption of a constant SFR over the past $100 \mathrm{Myr}$ is not likely to hold for our simulated galaxies, and we therefore may be overestimating their UV luminosities by a factor $\sim 2$ or so. A Kroupa IMF, on the other hand, would result in $\sim 1.7$ higher UV luminosity for a given SFR. We compare the simulated LFs to the Schechter function fits reported by B10 (shown in solid lines), as well as to these same relations corrected for dust extinction (dashed lines) by $(1.55,0.625,0.375,0,0)$ magnitudes at $z=(4,5,6,7,8)$ (cf. Table 8 in B10).

The KT07 LF exceeds the uncorrected B10 LF by $\sim 1$ dex at all redshifts less than 8 , and over the entire range of luminosities probed by B10. Applying the B10 dust correction brings the $z=4 \mathrm{LF}$ into agreement with the KT07 simulation. This agreement is remarkable, since it results without any tuning of our models. It is puzzling, however, since it seems to imply that there is no dwarf galaxy problem at $z=4$. In general, the steep faint-end LF slopes $(-1.7$ to -2.0$)$ at $z \geq 4$ reported by B10 appear to be in tension with the need to strongly suppressed star formation efficiency in low mass halos in order to match the lower redshift stellar mass functions and dwarf galaxy luminosity functions. Regardless of this, at $z>4$ the disagreement between our KT07 LFs and the dust-corrected observational ones remains substantial.

The KMT09_ZFz10 LF matches the KT07 one at high luminosities, reflecting the fact that $\mathrm{H}_{2}$-regulation is not effective in high mass (high $\mathrm{L}_{\mathrm{UV}}$ ) halos. Close to the sensitivity limit of the B10 observations, the KMT09_ZFz10 $\mathrm{LF}$ begins to roll over, improving the agreement between simulations and observations. This rollover, however, continues to lower luminosities, and it appears that in the KMT09_ZFz10 simulation the $\mathrm{H}_{2}$-suppression may be too efficient at low UV luminosities, at least if the faint end of the observed UV LF continues to rise to lower luminosities. As we discussed in $\S 4.2$, the exact mass scale of the SF suppression (and hence the downturn of the LF) is sensitive to the nature of the metal enrichment process, and also depends on the resolution of our simulations.

\subsection{Evolution of the Stellar Mass Density}

In Figure 19 we show the redshift evolution of the comoving stellar mass density (SMD) contributed by galaxies with $\mathrm{M}_{\star}>10^{7.75} \mathrm{M}_{\odot}$ in the KT07 and KMT09_ZFz10 simulations, compared to the observational determination from González et al. (2011). These authors used restframe optical photometry from Spitzer/IRAC to infer stellar masses for a sample of $\sim 300 z=4$ galaxies, and combined these with HST ACS and WFC3/IR restframe UV fluxes to establish an empirical stellar mass to UV luminosity relation. Assuming this same $\mathrm{M}_{\star}$ - $\mathrm{L}_{U V}$ extends to higher redshifts, they integrated the Bouwens et al. (2011) $z=4-7$ restframe UV luminosity function down to a limiting magnitude of $M_{\mathrm{UV}}=-18$ (corresponding to $\mathrm{M}_{\star}=10^{7.75} \mathrm{M}_{\odot}$ ), and obtained an empirical estimate of the SMD evolution: $\mathrm{SMD} \propto(1+z)^{-3.4 \pm 0.8}$, shown as the solid line and gray region in Figure 19.

To demonstrate the systematic uncertainty in this kind of empirical determination of the stellar density evolution, we also show results from Labbé et al. (2010a) (dotted line, SMD $\left.\propto(1+z)^{-6}\right)$ and Labbé et al. (2010b)

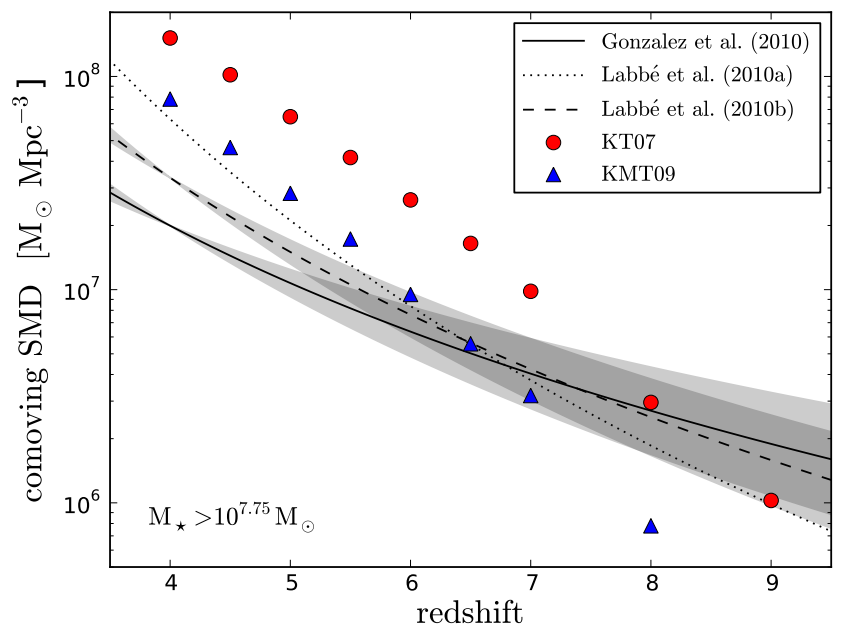

Figure 19. The stellar mass density for galaxies with $M_{\star}>$ $10^{7.75} \mathrm{M}_{\odot}$ vs. $\mathrm{z}$ in the KT07 and KMT09_ZFz10 simulations, compared to observational determinations from González et al. (2011) $\left(\mathrm{SMD} \propto(1+z)^{-3.4 \pm 0.8}\right.$, solid line), Labbé et al. (2010a), (SMD $\propto(1+z)^{-6}$, dotted line), and Labbé et al. (2010b) (SMD $\propto(1+z)^{-4.4 \pm 0.7}$, dashed line). The uncertainty in the slope reported by González et al. (2011) and Labbé et al. (2010b) is indicated by the gray regions, arbitrarily normalized at $z=4$.

(dashed line, SMD $\left.\propto(1+z)^{-4.4 \pm 0.7}\right)$, who performed similar work including $z=8$ Y-band dropouts, but used $\mathrm{M}_{\star}-\mathrm{L}_{\mathrm{UV}}$ relations derived from different low redshift data (Stark et al. 2009).

Likewise our simulated SMD's are not free from systematic uncertainties: on the one hand they are likely somewhat underestimated, especially at high redshift, since the simulations' limited box size $(12.5 \mathrm{Mpc})$ results in a delayed formation of massive halos, owing to the absence of density perturbations in the initial conditions with wavelengths exceeding the box size (Tormen \& Bertschinger 1996). On the other hand the lack of effective stellar feedback in our simulations probably artificially enhances the stellar mass density at all redshifts.

Given the substantial uncertainties in both observations and simulations, we consider the agreement between the two to be satisfactory. We do note, however, that the simulations prefer a somewhat steeper evolution of the SMD than González et al. (2011), and that the $\mathrm{H}_{2}{ }^{-}$ regulated SF reduces the SMD by a factor $\sim 3-5$ over the covered redshift range compared to the standard SF case. The difference between KT07 and KMT09_ZFz10 decreases towards lower redshifts, which can be attributed to the increase of the typical halo mass (the knee in the Press-Schechter massfunction) with decreasing redshift, resulting in an increasing fraction of resolved halos with mass above the $f_{\star}$-suppression mass scale.

\subsection{Evolution of the Star Formation Rate Density}

In Figure 20 we extend the comparison between our simulations and high-z observational data to the evolution of the SFR density, obtained by summing the SFR (Eq. 11) of all galaxies with $\mathrm{M}_{\star}>10^{7.75} \mathrm{M}_{\odot}\left(\mathrm{M}_{\mathrm{UV}}<\right.$ $-18)$ and dividing by the box volume. The observational data again comes from B10, who derived SFR densities by converting their restframe UV luminosities to SFR according to Madau et al. (1998), and integrated down to a limiting magnitude of $M_{\mathrm{UV}}=-18$, with (orange band) 


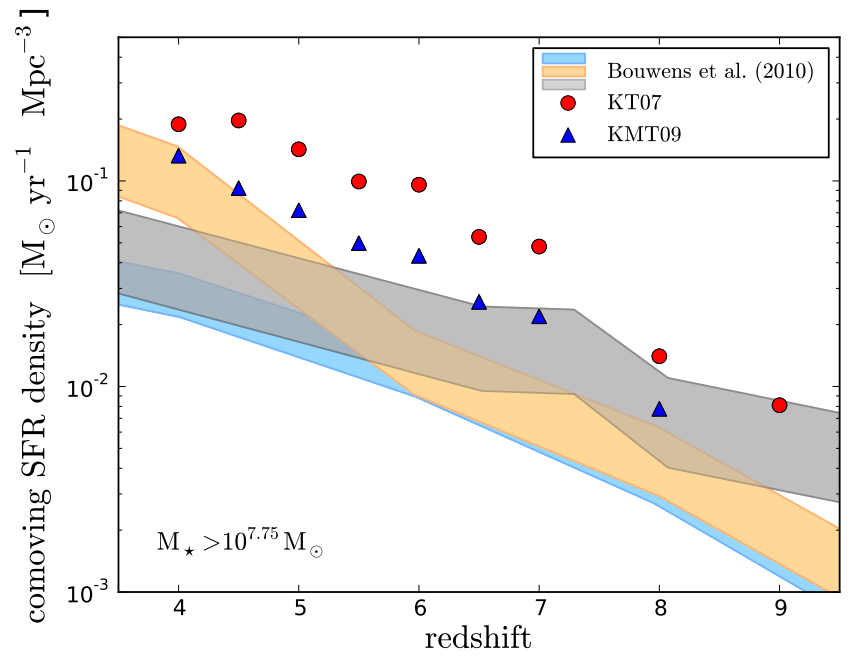

Figure 20. The SFR density for galaxies with $M_{\star}>10^{7.75} \mathrm{M}_{\odot}$ vs. $\mathrm{z}$ in the KT07 and KMT09_ZFz10 simulations, compared to observational determinations from Bouwens et al. (2011), which were obtained by converting restframe UV luminosities to SFR (Madau et al. 1998), without (blue band) and with dust corrections (orange band), and by converting stellar mass densities (Stark et al. 2009 Labbé et al. 2010a b González et al. 2011) to SFR densities (light gray band).

and without (blue band) a dust correction at $z \lesssim 6$ from Bouwens et al. (2009). Note that the very blue UV continuum slopes observed in $z \gtrsim 7$ galaxies (Bouwens et al. 2010, Bunker et al. 2010, Finkelstein et al. 2010, Oesch et al. 2010) imply low dust abundances and correspondingly weak extinction corrections at these high redshifts. For comparison we also show (light gray band) the SFR density implied by the SMD evolution discussed above, assuming a fixed SMD $\propto(1+z)^{-4.4}$ extrapolation to $z \gtrsim 8$ (Labbé et al. 2010b).

Our simulated SFR densities are somewhat higher than those reported by Bouwens et al. (2011). $\mathrm{H}_{2}$-regulated $\mathrm{SF}$, however, reduces the SFR densities by about a factor of two, and brings them close to agreement with those determined from the SMD evolution, and only a factor $\sim 3$ above the luminosity density derived values at $z>6$. At lower redshifts, the rise due to dust corrections in the observational SFR densities reduces the difference, and an extrapolation of the KMT09_ZFz10 SFR densities to $z=4$ is in good agreement with the dust corrected observational determination. If the observed steep UV continuum slopes are a selection effect and not representative of typical $z \gtrsim 7$ galaxies, then a more heavily dust obscured population of galaxies that has been missed by current surveys could close the high redshift gap between our simulations and current data. Additionally it is possible that a more effective stellar feedback implementation in our simulations could further reduce the simulated SFR densities.

\section{SUMMARY AND DISCUSSION}

Motivated by the observational fact that star formation, both in the local universe (e.g. Bigiel et al. 2008) and at intermediate redshifts (e.g. Genzel et al.|2010), correlates more tightly with the density of gas in the molecular phase than the total gas density, we have implemented an $\mathrm{H}_{2}$-regulated $\mathrm{SF}$ prescription in cosmological galaxy formation simulations, with the goal of inves- tigating whether this new ingredient can help to alleviate outstanding problems in our theoretical understanding of dwarf galaxy formation.

Rather than following the non-equilibrium molecular hydrogen chemistry including time-dependent and spatially inhomogeneous radiation transfer of the ionizing and $\mathrm{H}_{2}$-dissociating stellar radiation field (G09), we utilize in our simulations the results of one-dimensional radiative transfer calculations of the $\mathrm{H}_{2}$ formationdissociation balance in an idealized spherical giant atomic-molecular complex subject to a uniform and isotropic Lyman-Werner (LW) radiation field (Krumholz et al. 2008, 2009, McKee \& Krumholz 2010). These calculations showed that under the assumption of two-phase equilibrium in the ISM, the $\mathrm{H}_{2}$ abundance is determined entirely by the column density and metallicity of gas on $\sim 100$ pc scales - comparable to the cell size of our highest resolution grid cells.

We have deliberately chosen a weak implementation of supernova feedback in order to isolate the effects of the molecular hydrogen chemistry on star formation in a cosmological setting. Remarkably, we find that accounting for the transition from $\mathrm{HI}$ to $\mathrm{H}_{2}$ alone is able to fulfill one of the functions of the neglected supernova feedback, namely the suppression of star formation in low mass halos. The main results of our study are summarized as follows.

1. Both our conventional and $\mathrm{H}_{2}$-regulated SF prescriptions are able to reproduce the observational scaling relation between the SFR surface density and the total gas density, the Kennicutt-Schmidt relation (Fig. 3).

2. With the conventional SF prescription the observed turnover of $\Sigma_{\mathrm{SFR}}$ at low $\Sigma_{\text {gas }}$ is recovered by manually tuning a SF threshold density. The $\mathrm{H}_{2}$ regulated prescription, however, automatically reproduces this cutoff (Fig. 5) and thus reduces the number of free parameters in the SF prescription by one.

3. We are able to reproduce many of the observational results pertaining to the $\mathrm{KS}$ relation, for example the slope of the molecular gas KS relation $\left(\Sigma_{\mathrm{SFR}}-\Sigma_{\mathrm{H}_{2}}\right)$ and the much weaker correlation of $\Sigma_{\mathrm{SFR}}$ with the atomic gas surface density (Fig. 6). We also recover the observed metallicity dependence of the low $\Sigma_{\text {gas }}$ turnover in the KS relation (Fig. 7), which occurs at higher $\Sigma_{\text {gas }}$ in lower metallicity systems, reflecting the atomic-tomolecular gas transition. Lastly, in agreement with recent observations, we see an increased scatter in the $\mathrm{H}_{2} \mathrm{KS}$ relation for smaller spatial smoothing scales.

4. We expect an increased scatter in the KS relation at higher redshifts, since the typically larger stellar velocity dispersions in high redshift systems will allow young stars to wander outside of high column density regions.

5. $\mathrm{H}_{2}$-regulation suppresses $\mathrm{SF}$ in low mass halos, reducing the need for stellar feedback (Fig. 11), as previously reported by G09 and GK10 for a set of 
cosmological zoom-in galaxy formation simulations including non-equilibrium $\mathrm{H}_{2}$ formation and radiative transfer. We confirm these prior results with better statistics (hundreds of halos) and without the need for a complicated and expensive radiative transfer treatment.

6. The halo mass dependence appears to be tied primarily to a difference in metal enrichment, rather than gas column density (Fig. 14). On the other hand, for a given metallicity floor (set for example by the first generation of Pop.III supernovae), the star forming ability of halos is determined by the highest column densities their gas can condense to. Low mass halos don't have sufficiently high column density gas to allow the transition to molecular phase and hence star formation.

7. Suppressing the star formation efficiency in low mass halos lowers the cosmic stellar mass density and star formation rate density. We find reasonable agreement between our $\mathrm{H}_{2}$-regulated simulation and observational determinations of the evolution of the SMD and SFR-density at $z>4$. Both simulations and observations are subject to large systematic uncertainties.

As we discussed throughout the text, a number of caveats apply to our findings. First, the results we have presented here are not completely independent of the implementation details of our simulations (e.g. 2-phase equilibrium assumption, metallicity floor, numerical resolution). We therefore cannot claim with any confidence to have pinpointed the halo mass below which star formation is suppressed due to the inability of gas to become molecular.

Second, given our computational resources, we were unable to continue our simulations beyond $z=4$. It is possible that a large build-up of atomic gas will lead to a burst of star formation at lower redshift $(z \simeq 1-3)$, when metallicities have increased sufficiently to allow the transition to $\mathrm{H}_{2}$ to occur, and in fact this may help to explain how the specific SFR of $10^{10}-10^{12} \mathrm{M}_{\odot}$ halos can exceed the instantaneous gas accretion rate at these redshifts (Krumholz \& Dekel 2011).

Lastly, like most cosmological galaxy formation simulations to date, our simulated galaxies suffer from the socalled "baryonic overcooling problem", resulting in unrealistically high central densities (and hence strongly peaked circular velocity curves) and stellar mass fractions in our high mass halos that are too large compared to observations. Although we cannot exclude the possibility that these artificially high central densities affect the results of our study, we believe that this makes our results conservative, in the sense that a more realistic simulation with lower central gas densities would likely experience even more $\mathrm{H}_{2}$-regulated SF suppression. Reduced central densities would demand higher metallicity for the transition to $\mathrm{H}_{2}$ to occur, and at the same time they would allow the metals ejected by stars to become diluted more easily, thereby reducing the metallicity of the high density star-forming gas.

Even allowing for these caveats, we believe that the atomic-to-molecular gas transition may play an important role in regulating star formation in low mass halos.
It may also help to explain the result recently reported by Boylan-Kolchin et al. (2011) that collisionless simulations of galactic dark matter substructure (Via Lactea II and Aquarius) predict a number of subhalos too centrally concentrated to host any of the known Milky Way dwarf satellite galaxies. As Boylan-Kolchin et al. (2011) suggest, one possible explanation of this puzzle is that star formation in dark matter halos becomes stochastic below some mass. The sensitivity of an $\mathrm{H}_{2}$-regulated $\mathrm{SF}$ prescription to early metal enrichment may provide the necessary stochasticity.

In our simulations, $\mathrm{H}_{2}$-regulation quenches $\mathrm{SF}$ in dwarf galaxies at the outset, without the need to artificially enhance stellar feedback by turning off gas cooling or hydrodynamically decoupling momentum-driven winds, as is commonly done in the literature. These latter implementations of enhanced stellar feedback quench SF in dwarf galaxies due to the inability of their shallow potential wells to retain the resulting gas outflows. In $\mathrm{H}_{2}$ regulated SF, by contrast, it is the inability of low mass halos to accumulate a sufficiently large column density of metal-enriched material that results in suppressed SF. Efficient supernova feedback implies the presence of a large pool of hot gas as well as galactic winds, neither of which are necessarily present in an $\mathrm{H}_{2}$-regulated scenario. This difference may be be a good way to observationally distinguish the two mechanisms.

Taken at face value, our results imply that many low mass dark matter halos at high redshift should be filled with relatively cold, yet atomic gas, which is prevented from becoming molecular by its low metallicity. While neutral gas surveys with radio telescopes are hard pressed to reach beyond the local universe, constraints on the HI mass function do exist at low redshift. The ALFALFA survey (Martin et al. 2010), for example, has reported a $z \lesssim 0.06 \mathrm{HI}$ mass function down to $\log \left(M_{\mathrm{HI}} / \mathrm{M}_{\odot}\right)=6.2$, that is well fit by a Schechter function with a low mass slope of $\alpha=-1.33$. This is considerably shallower than the mass function of dark matter halos $(\alpha \approx-1.8$, Boylan-Kolchin et al. 2009), indicating that not all low mass dark matter halos can be allowed to retain their full baryonic content in the form of cold, atomic gas down to the present epoch. Another constraint may come from the statistics of damped Lyman- $\alpha$ (DLA) systems at $z \lesssim 5$ (Prochaska \& Wolfe 2009, Noterdaeme et al. 2009). Whether the observed frequency distribution of DLA column densities and their covering fraction is consistent with large amounts of atomic gas in high redshift dwarf galaxies should be investigated in detail.

Strongly suppressing star formation in low mass halos at high redshift could make it difficult for faint galaxies to reionize the universe by $z=6$, as is commonly advocated (e.g. Madau et al. 1999). Indeed, in our simulations the total number of hydrogen ionizing photons per hydrogen atom ${ }^{5}$ produced by $z=6$ is reduced from $\sim 18$ in KT07 to $\sim 6$ in KMT09. As shown by (Kuhlen \& FaucherGiguere 2012), a suppression scale of $\mathrm{M}_{\mathrm{h}}=10^{10} \mathrm{M}_{\odot}$ can simultaneously satisfy reionization and lower redshift Lyman- $\alpha$ forest constraints only if the escape frac-

\footnotetext{
${ }^{5}$ We assume 4000 hydrogen ionizing photons per stellar baryon for every solar mass of stars formed, as appropriate for a Salpeter IMF from 0.1 to $125 \mathrm{M}_{\odot}$ and a mean ionizing photon energy of 20 eV (Madau et al. 1999) Leitherer et al. 1999).
} 
tion of ionizing radiation evolves strongly from $z=4$ to higher redshifts. A slightly smaller suppression mass of $\sim 10^{9} \mathrm{M}_{\odot}$ (e.g. as in Krumholz \& Dekel 2011), however, is in good agreement with all constraints.

While we have emphasized in this work that $\mathrm{H}_{2}$ regulated SF can perform some of the functions typically assigned to supernova feedback, we of course acknowledge that supernovae do in fact occur in nature and that their associated injection of energy and momentum into the surrounding interstellar medium is likely to significantly impact subsequent star formation, and may help to solve the problem of forming galaxies that are too concentrated in the simulations. Future research will be necessary to elucidate how the interplay of molecular chemistry and supernova feedback shapes star formation in dwarf galaxies and beyond.

MK acknowledges stimulating and fruitful discussions with F. Bigiel, C.-A. Faucher-Giguère, R. Feldmann, N. Gnedin, P. Hopkins, D. Kereš, A. Kravtsov, M. McQuinn, N. Murray, M. Norman, S. Skory, and E. Quataert. We thank A. Bolatto for providing us with a table of $\mathrm{H}_{2}$ and $\mathrm{HI}$ column densities towards the SMC. MRK acknowledges support from: an Alfred P. Sloan Fellowship; the NSF through grants AST0907739 and CAREER-0955300; and NASA through Astrophysics Theory and Fundamental Physics grant NNX09AK31G and through a Spitzer Space Telescope Theoretical Research Program grant. PM acknowledges support from NASA through grant NNX09AJ34G and from NSF through grant AST-0908910. JHW is supported by NASA through Hubble Fellowship grant \#1206370 awarded by the Space Telescope Science Institute, which is operated by the Association of Universities for Research in Astronomy, Inc., for NASA, under contract NAS 5-26555.

A special thank you to all Enzo developers for being such a great community and contributing your time and expertise to the development and improvement of the Enzo code. Analysis of the simulations was greatly aided by the yt software (Turk et al. 2011).

\section{REFERENCES}

Abel, T., Anninos, P., Zhang, Y., \& Norman, M. L. 1997, New Astronomy, 2, 181

Agertz, O., Teyssier, R., \& Moore, B. 2009, Monthly Notices of the Royal Astronomical Society, 397, L64

—. 2011, Monthly Notices of the Royal Astronomical Society, 410, 1391

Anninos, P., Zhang, Y., Abel, T., \& Norman, M. L. 1997, New Astronomy, 2, 209

Avila-Reese, V., Colín, P., González-Samaniego, A., Valenzuela, O., Firmani, C., Velázquez, H., \& Ceverino, D. 2011, The Astrophysical Journal, 736, 134

Behroozi, P. S., Conroy, C., \& Wechsler, R. H. 2010, The Astrophysical Journal, 717, 379

Benson, A. J., Bower, R. G., Frenk, C. S., Lacey, C. G., Baugh, C. M., \& Cole, S. 2003, The Astrophysical Journal, 599, 38

Benson, A. J., Frenk, C. S., Lacey, C. G., Baugh, C. M., \& Cole, S. 2002, Monthly Notices of the Royal Astronomical Society, 333,177

Bigiel, F., Leroy, A., Walter, F., Brinks, E., de Blok, W. J. G., Madore, B., \& Thornley, M. D. 2008, The Astronomical Journal, 136, 2846

Blitz, L. 1993, in Protostars and Planets III, 125-161
Blumenthal, G. R., Faber, S. M., Primack, J. R., \& Rees, M. J. 1984, Nature, 311, 517

Bolatto, A. D., Leroy, A. K., Rosolowsky, E., Walter, F., \& Blitz, L. 2008, The Astrophysical Journal, 686, 948

Bolatto, A. D., et al. 2011, ArXiv e-print 1107.1717

Bouwens, R. J., Illingworth, G. D., Franx, M., \& Ford, H. 2007,

The Astrophysical Journal, 670, 928

Bouwens, R. J., et al. 2009, The Astrophysical Journal, 705, 936

—. 2010, The Astrophysical Journal Letters, 708, L69

- 2011, The Astrophysical Journal, 737, 90

Boylan-Kolchin, M., Bullock, J. S., \& Kaplinghat, M. 2011,

Monthly Notices of the Royal Astronomical Society, 415, L40

Boylan-Kolchin, M., Springel, V., White, S. D. M., Jenkins, A., \& Lemson, G. 2009, Monthly Notices of the Royal Astronomical Society, 398, 1150

Brook, C. B., Stinson, G., Gibson, B. K., Wadsley, J., \& Quinn, T. 2012, ArXiv e-print 1201.3359

Bryan, G. L., \& Norman, M. L. 1998, The Astrophysical Journal, 495, 80

Bryan, G. L., Norman, M. L., Stone, J. M., Cen, R., \& Ostriker, J. P. 1995, Computer Physics Communications, 89, 149

Bullock, J. S., Kravtsov, A. V., \& Weinberg, D. H. 2000, The Astrophysical Journal, 539, 517

Bunker, A. J., et al. 2010, Monthly Notices of the Royal Astronomical Society, 409, 855

Cen, R., \& Ostriker, J. P. 1993, The Astrophysical Journal, 417, 404

—. 2006, The Astrophysical Journal, 650, 560

Ceverino, D., \& Klypin, A. 2009, The Astrophysical Journal, 695, 292

Choi, J., \& Nagamine, K. 2011, ArXiv e-prints 1101.5656

Cirasuolo, M., McLure, R. J., Dunlop, J. S., Almaini, O.,

Foucaud, S., \& Simpson, C. 2010, Monthly Notices of the Royal Astronomical Society, 401, 1166

Cole, S., Lacey, C. G., Baugh, C. M., \& Frenk, C. S. 2000, Monthly Notices of the Royal Astronomical Society, 319, 168

Colella, P., \& Woodward, P. R. 1984, Journal of Computational Physics, 54, 174

Colín, P., Avila-Reese, V., Vázquez-Semadeni, E., Valenzuela, O., \& Ceverino, D. 2010, The Astrophysical Journal, 713, 535

Conroy, C., \& Wechsler, R. H. 2009, The Astrophysical Journal, 696, 620

Cresci, G., et al. 2009, The Astrophysical Journal, 697, 115

Daddi, E., et al. 2010, The Astrophysical Journal, 714, L118

Dekel, A., \& Silk, J. 1986, The Astrophysical Journal, 303, 39

del P. Lagos, C., Lacey, C. G., Baugh, C. M., Bower, R. G., \& Benson, A. J. 2010, ArXiv e-print 1011.5506

Diemand, J., Kuhlen, M., Madau, P., Zemp, M., Moore, B., Potter, D., \& Stadel, J. 2008, Nature, 454, 735

D’Onghia, E., Besla, G., Cox, T. J., \& Hernquist, L. 2009, Nature, 460, 605

Draine, B. T. 1978, The Astrophysical Journal Supplement Series, 36,595

Efstathiou, G. 1992, Monthly Notices of the Royal Astronomical Society, 256, 43P

-. 2000, Monthly Notices of the Royal Astronomical Society, 317,697

Eisenstein, D. J., \& Hu, W. 1999, The Astrophysical Journal, 511,5

Eisenstein, D. J., \& Hut, P. 1998, The Astrophysical Journal, 498, 137

Fall, S. M., Krumholz, M. R., \& Matzner, C. D. 2010, The Astrophysical Journal, 710, L142

Faucher-Giguère, C., \& Kereš, D. 2011, Monthly Notices of the Royal Astronomical Society, 412, L118

Faucher-Giguère, C., Kereš, D., \& Ma, C. 2011, Monthly Notices of the Royal Astronomical Society, 417, 2982

Faucher-Giguère, C., Lidz, A., Zaldarriaga, M., \& Hernquist, L. 2009, The Astrophysical Journal, 703, 1416

Feldmann, R., \& Gnedin, N. Y. 2011, The Astrophysical Journal, 727, L12

Feldmann, R., Gnedin, N. Y., \& Kravtsov, A. V. 2011, The Astrophysical Journal, 732, 115

Ferland, G. J., Korista, K. T., Verner, D. A., Ferguson, J. W., Kingdon, J. B., \& Verner, E. M. 1998, Publications of the Astronomical Society of the Pacific, 110, 761 
Finkelstein, S. L., Papovich, C., Giavalisco, M., Reddy, N. A., Ferguson, H. C., Koekemoer, A. M., \& Dickinson, M. 2010, The Astrophysical Journal, 719, 1250

Font, A. S., et al. 2011, ArXiv e-prints 1103.0024

Fontanot, F., Lucia, G. D., Monaco, P., Somerville, R. S., \& Santini, P. 2009, Monthly Notices of the Royal Astronomical Society, 397, 1776

Fu, J., Guo, Q., Kauffmann, G., \& Krumholz, M. R. 2010, Monthly Notices of the Royal Astronomical Society, 409, 515

Genel, S., et al. 2010, ArXiv e-prints 1011.0433

Genzel, R., et al. 2010, Monthly Notices of the Royal Astronomical Society, 407, 2091

Giavalisco, M., et al. 2004, The Astrophysical Journal, 600, L93

Gnedin, N. Y. 1998, Monthly Notices of the Royal Astronomical Society, 294, 407

Gnedin, N. Y., \& Kravtsov, A. V. 2010, The Astrophysical Journal, 714, 287

- 2011, The Astrophysical Journal, 728, 88

Gnedin, N. Y., Kravtsov, A. V., \& Rudd, D. H. 2011, The Astrophysical Journal Supplement Series, 194, 46

Gnedin, N. Y., Tassis, K., \& Kravtsov, A. V. 2009, The Astrophysical Journal, 697, 55

Gnedin, O. Y., Hernquist, L., \& Ostriker, J. P. 1999, The Astrophysical Journal, 514, 109

Goldbaum, N. J., Krumholz, M. R., Matzner, C. D., \& McKee, C. F. 2011, ArXiv e-print 1105.6097

González, V., Labbé, I., Bouwens, R. J., Illingworth, G., Franx, M., \& Kriek, M. 2011, The Astrophysical Journal, 735, L34

Governato, F., et al. 2010, Nature, 463, 203

Guedes, J., Callegari, S., Madau, P., \& Mayer, L. 2011, ArXiv e-prints 1103.6030

Guo, Q., White, S., Li, C., \& Boylan-Kolchin, M. 2010, Monthly Notices of the Royal Astronomical Society, 404, 1111

Haardt, F., \& Madau, P. 2001, in Clusters of Galaxies and the High Redshift Universe Observed in X-rays, 64

Hopkins, P. F., Quataert, E., \& Murray, N. 2011, ArXiv e-print 1101.4940

Katz, N. 1992, The Astrophysical Journal, 391, 502

Kauffmann, G., White, S. D. M., \& Guiderdoni, B. 1993, Monthly Notices of the Royal Astronomical Society, 264, 201

Kennicutt, R. C. 1998, The Astrophysical Journal, 498, 541

Kennicutt, R. C., et al. 2007, The Astrophysical Journal, 671, 333

Klypin, A., Kravtsov, A. V., Valenzuela, O., \& Prada, F. 1999, The Astrophysical Journal, 522, 82

Komatsu, E., et al. 2011, The Astrophysical Journal Supplement Series, 192, 18

Kravtsov, A. V. 2003, The Astrophysical Journal, 590, L1

Krumholz, M., \& Burkert, A. 2010, The Astrophysical Journal, 724,895

Krumholz, M. R., \& Dekel, A. 2011, ArXiv e-prints 1106.0301

Krumholz, M. R., \& Gnedin, N. Y. 2011, The Astrophysical Journal, 729, 36

Krumholz, M. R., Leroy, A. K., \& McKee, C. F. 2011, The Astrophysical Journal, 731, 25

Krumholz, M. R., \& Matzner, C. D. 2009, The Astrophysical Journal, 703, 1352

Krumholz, M. R., Matzner, C. D., \& McKee, C. F. 2006, The Astrophysical Journal, 653, 361

Krumholz, M. R., McKee, C. F., \& Tumlinson, J. 2008, The Astrophysical Journal, 689, 865

_. 2009, The Astrophysical Journal, 693, 216

Krumholz, M. R., \& Tan, J. C. 2007, The Astrophysical Journal, 654,304

Kuhlen, M., \& Faucher-Giguere, C. 2012, ArXiv e-prints 1201.0757

Labbé, I., et al. 2010a, The Astrophysical Journal, 708, L26

- 2010b, The Astrophysical Journal, 716, L103

Larson, R. B. 1974, Monthly Notices of the Royal Astronomical Society, 169, 229

Leitherer, C., et al. 1999, The Astrophysical Journal Supplement Series, 123, 3

Leroy, A. K., Walter, F., Brinks, E., Bigiel, F., de Blok, W. J. G., Madore, B., \& Thornley, M. D. 2008, The Astronomical Journal, 136, 2782

Liu, G., Koda, J., Calzetti, D., Fukuhara, M., \& Momose, R. 2011, ArXiv e-print 1104.4122
Machacek, M. E., Bryan, G. L., \& Abel, T. 2001, The Astrophysical Journal, 548, 509

Madau, P., Diemand, J., \& Kuhlen, M. 2008, The Astrophysical Journal, 679, 1260

Madau, P., Haardt, F., \& Rees, M. J. 1999, The Astrophysical Journal, 514, 648

Madau, P., Pozzetti, L., \& Dickinson, M. 1998, The Astrophysical Journal, 498, 106

Marchesini, D., van Dokkum, P. G., Schreiber, N. M. F., Franx, M., Labbé, I., \& Wuyts, S. 2009, The Astrophysical Journal, 701, 1765

Martin, A. M., Papastergis, E., Giovanelli, R., Haynes, M. P., Springob, C. M., \& Stierwalt, S. 2010, The Astrophysical Journal, 723, 1359

Matzner, C. D. 2002, The Astrophysical Journal, 566, 302

Mayer, L., Mastropietro, C., Wadsley, J., Stadel, J., \& Moore, B. 2006, Monthly Notices of the Royal Astronomical Society, 369, 1021

McKee, C. F. 1989, The Astrophysical Journal, 345, 782

McKee, C. F., \& Krumholz, M. R. 2010, The Astrophysical Journal, 709, 308

Moore, B., Ghigna, S., Governato, F., Lake, G., Quinn, T., Stadel, J., \& Tozzi, P. 1999, The Astrophysical Journal, 524, L19

Moster, B. P., Somerville, R. S., Maulbetsch, C., van den Bosch,

F. C., Macciò, A. V., Naab, T., \& Oser, L. 2010, The Astrophysical Journal, 710, 903

Murray, N., Quataert, E., \& Thompson, T. A. 2010, The Astrophysical Journal, 709, 191

Nagamine, K., Ostriker, J. P., Fukugita, M., \& Cen, R. 2006, The Astrophysical Journal, 653, 881

Nakamura, F., \& Li, Z. 2007, The Astrophysical Journal, 662, 395

Norman, C., \& Silk, J. 1980, The Astrophysical Journal, 238, 158

Noterdaeme, P., Petitjean, P., Ledoux, C., \& Srianand, R. 2009, Astronomy and Astrophysics, 505, 1087

Ocvirk, P., Pichon, C., \& Teyssier, R. 2008, Monthly Notices of the Royal Astronomical Society, 390, 1326

Oesch, P. A., et al. 2010, The Astrophysical Journal, 709, L16

Oppenheimer, B. D., \& Davé, R. 2006, Monthly Notices of the Royal Astronomical Society, 373, 1265

Oppenheimer, B. D., Davé, R., Kereš, D., Fardal, M., Katz, N., Kollmeier, J. A., \& Weinberg, D. H. 2010, Monthly Notices of the Royal Astronomical Society, 406, 2325

O'Shea, B. W., Nagamine, K., Springel, V., Hernquist, L., \& Norman, M. L. 2005, The Astrophysical Journal Supplement Series, 160, 1

Piontek, F., \& Steinmetz, M. 2011, Monthly Notices of the Royal Astronomical Society, 410, 2625

Prochaska, J. X., \& Wolfe, A. M. 2009, The Astrophysical Journal, 696, 1543

Rafelski, M., Wolfe, A. M., \& Chen, H. 2011, The Astrophysical Journal, 736, 48

Rafelski, M., Wolfe, A. M., Cooke, J., Chen, H., Armandroff, T. E., \& Wirth, G. D. 2009, The Astrophysical Journal, 703 , 2033

Rashkov, V., Madau, P., Kuhlen, M., \& Diemand, J. 2012, The Astrophysical Journal, 745, 142

Robertson, B. E., \& Kravtsov, A. V. 2008, The Astrophysical Journal, 680, 1083

Rodríguez, M., \& Delgado-Inglada, G. 2011, The Astrophysical Journal, 733, L50

Sales, L. V., Navarro, J. F., Schaye, J., Vecchia, C. D., Springel, V., \& Booth, C. M. 2010, Monthly Notices of the Royal Astronomical Society, 409, 1541

Schaye, J., et al. 2010, Monthly Notices of the Royal Astronomical Society, 402, 1536

Schruba, A., Leroy, A. K., Walter, F., Sandstrom, K., \& Rosolowsky, E. 2010, The Astrophysical Journal, 722, 1699

Sheth, R. K., \& Tormen, G. 1999, Monthly Notices of the Royal Astronomical Society, 308, 119

Smith, B., Sigurdsson, S., \& Abel, T. 2008, Monthly Notices of the Royal Astronomical Society, 385, 1443

Smith, B. D., Hallman, E. J., Shull, J. M., \& O'Shea, B. W. 2011, The Astrophysical Journal, 731, 6

Somerville, R. S., Hopkins, P. F., Cox, T. J., Robertson, B. E., \& Hernquist, L. 2008, Monthly Notices of the Royal Astronomical Society, 391, 481 
Springel, V., Frenk, C. S., \& White, S. D. M. 2006, Nature, 440, 1137

Springel, V., \& Hernquist, L. 2003, Monthly Notices of the Royal Astronomical Society, 339, 289

Springel, V., et al. 2008, Monthly Notices of the Royal Astronomical Society, 391, 1685

Stadel, J., Potter, D., Moore, B., Diemand, J., Madau, P., Zemp, M., Kuhlen, M., \& Quilis, V. 2009, Monthly Notices of the Royal Astronomical Society, 398, L21

Stark, D. P., Ellis, R. S., Bunker, A., Bundy, K., Targett, T., Benson, A., \& Lacy, M. 2009, The Astrophysical Journal, 697, 1493

Stinson, G., Seth, A., Katz, N., Wadsley, J., Governato, F., \& Quinn, T. 2006, Monthly Notices of the Royal Astronomical Society, 373, 1074

Thacker, R. J., \& Couchman, H. M. P. 2000, The Astrophysical Journal, 545, 728

Tormen, G., \& Bertschinger, E. 1996, The Astrophysical Journal, 472,14

Toro, E. F., Spruce, M., \& Speares, W. 1994, Shock Waves, 4, 25

Turk, M. J., Smith, B. D., Oishi, J. S., Skory, S., Skillman, S. W., Abel, T., \& Norman, M. L. 2011, The Astrophysical Journal Supplement Series, 192, 9
Wang, J., Lucia, G. D., Kitzbichler, M. G., \& White, S. D. M. 2008, Monthly Notices of the Royal Astronomical Society, 384, 1301

Wang, P., Li, Z., Abel, T., \& Nakamura, F. 2010, The Astrophysical Journal, 709, 27

White, S. D. M., \& Rees, M. J. 1978, Monthly Notices of the Royal Astronomical Society, 183, 341

Wise, J. H., Turk, M. J., Norman, M. L., \& Abel, T. 2012, The Astrophysical Journal, 745, 50

Wolfe, A. M., \& Chen, H. 2006, The Astrophysical Journal, 652, 981

Wolfire, M. G., McKee, C. F., Hollenbach, D., \& Tielens, A. G. G. M. 2003, The Astrophysical Journal, 587, 278

Wolfire, M. G., Tielens, A. G. G. M., Hollenbach, D., \& Kaufman, M. J. 2008, The Astrophysical Journal, 680, 384

Wong, T., \& Blitz, L. 2002, The Astrophysical Journal, 569, 157

Yepes, G., Kates, R., Khokhlov, A., \& Klypin, A. 1997, Monthly Notices of the Royal Astronomical Society, 284, 235

Zheng, Z., Coil, A. L., \& Zehavi, I. 2007, The Astrophysical Journal, 667, 760

APPENDIX

SOBOLEV-LIKE APPROXIMATION OF $\Sigma$
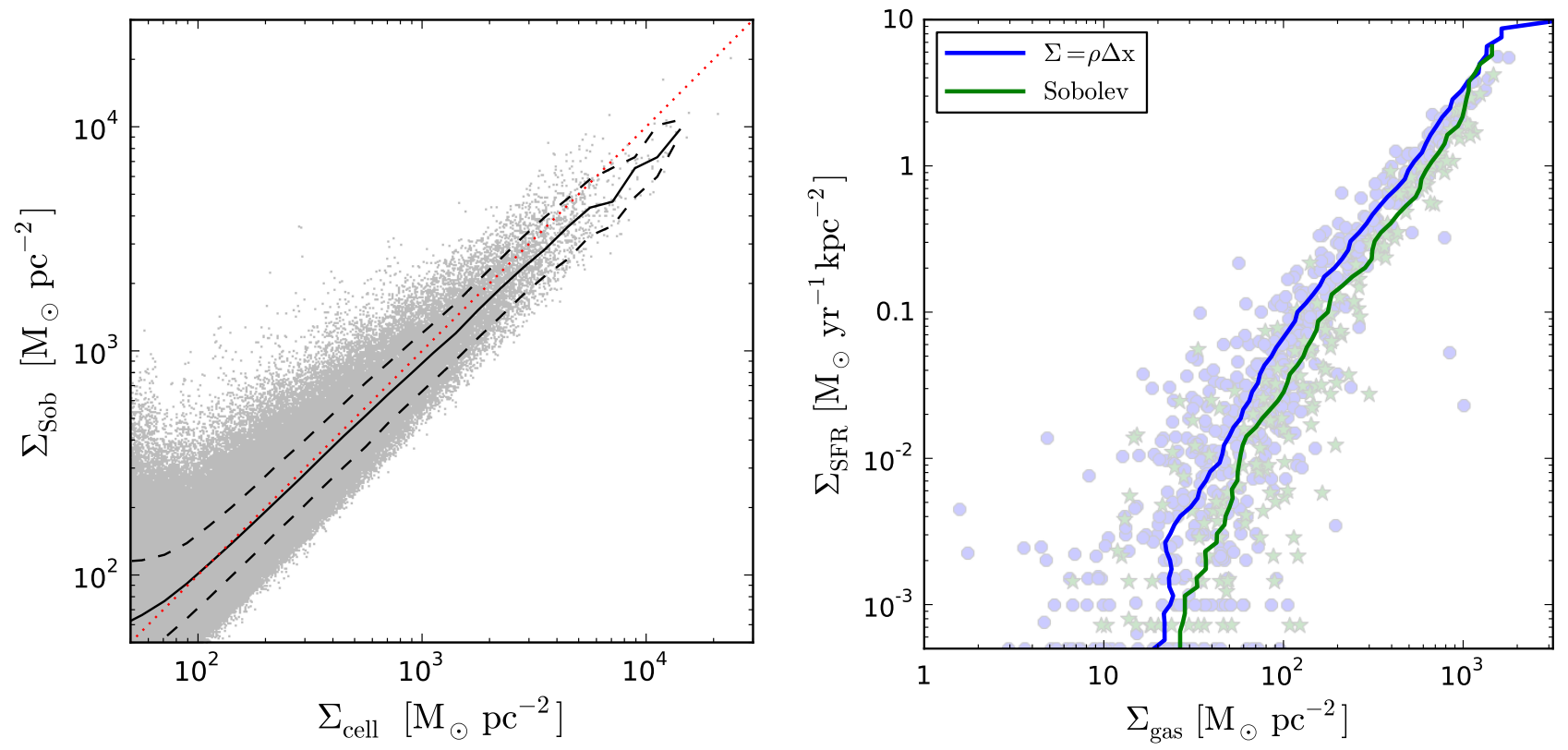

Figure 21. Left: Comparison of a Sobolev-like estimate of the column density $\left(\Sigma_{\text {Sob }}=\rho \times(\rho / \nabla \rho)\right.$, see text for details $)$ with the simple grid-cell column $\left(\Sigma_{\text {cell }}=\rho \Delta x\right)$, obtained by post-processing the $z=4$ KMT09 output. Only columns greater than $50 \mathrm{M}_{\odot} \mathrm{pc}^{-2}$ are shown, since the $\mathrm{H}_{2}$ content is negligible at lower columns. The solid and dashed black lines show the median and $16^{\text {th }}-84^{\text {th }}$ percentiles of $\Sigma_{\text {Sob }}$ for a given $\Sigma_{\text {cell. }}$. The Sobolev-like estimate results in slightly lower columns, especially at high columns. Right: KS relation for simulation KMT09_Sob, in which the Sobolev-like approximation was used at simulation run-time to calculate the column density entering the KMT09 expression for $f_{\mathrm{H}_{2}}$. Note that we did not use the Sobolev-like approximation to determine the x-axis quantity $\Sigma_{\text {gas }}$, the column smoothed at $l=3(\sim 1 \mathrm{kpc})$. The slightly lower columns with the Sobolev-like approximation result in lower $f_{\mathrm{H}_{2}}$ and hence a slightly reduced SFR at a given $\Sigma_{\text {gas. }}$.

One way to alleviate the explicit resolution dependence in our implementation of the KMT09 algorithm (Eq. 449 is to use a Sobolev-like approximation of the column density (see Gnedin et al. 2009), instead of simply multiplying the grid cell density by its width. We have implemented a Sobolev-like approximation as follows:

$$
\begin{aligned}
\rho_{x}^{+} & =0.5\left(\rho_{i, j, k}+\rho_{i+1, j, k}\right), & \rho_{x}^{-} & =0.5\left(\rho_{i, j, k}+\rho_{i-1, j, k}\right), \\
h_{x}^{+} & =\frac{\rho_{x}^{+}}{\left|\rho_{i+1, j, k}-\rho_{i, j, k}\right| / \Delta x}, & h_{x}^{-} & =\frac{\rho_{x}^{-}}{\left|\rho_{i, j, k}-\rho_{i-1, j, k}\right| / \Delta x}, \\
\Sigma_{x}^{+} & =\rho_{x}^{+} \cdot h_{x}^{+}, & \Sigma_{x}^{-} & =\rho_{x}^{-} \cdot h_{x}^{-},
\end{aligned}
$$


and similarly for the $\mathrm{y}$ and $\mathrm{z}$ directions. This definition has the virtue that it is resolution-independent, at least to the extent that the density field itself is resolution-independent. To obtain a total column density, we take the harmonic mean over the six cardinal directions,

$$
\langle\Sigma\rangle=\frac{6}{1 / \Sigma_{x}^{+}+1 / \Sigma_{x}^{-}+1 / \Sigma_{y}^{+}+1 / \Sigma_{y}^{-}+1 / \Sigma_{z}^{+}+1 / \Sigma_{z}^{-}} .
$$

We have applied this Sobolev-like approximation for $\Sigma_{\text {gas }}$ in a post-processing analysis of the $z=4$ output of the KMT09 simulation, and compared the resulting column density with the simple grid cell based estimate used in the simulation, see the left panel of Fig. 21. We're only showing points with $\Sigma>50 \mathrm{M}_{\odot} \mathrm{pc}^{-2}$, since at lower columns the $\mathrm{H}_{2}$ abundance, and hence SFR, is negligible. The median of $\Sigma_{\text {Sob }}$ lies close to the 1-1 line, but drops below it around $\Sigma_{\text {cell }}=100 \mathrm{M}_{\odot} \mathrm{pc}^{-2}$, implying that the Sobolev-like approximation systematically yields slightly lower values at high $\Sigma_{\text {cell }}$. At $\Sigma_{\text {cell }}=100,1000$, and $5000 \mathrm{M}_{\odot} \mathrm{pc}^{-2}$ the median of $\Sigma_{\text {Sob }}$ is 101,880 , and $3930 \mathrm{M}_{\odot} \mathrm{pc}^{-2}$, respectively. The $1-\sigma$ scatter of $\Sigma_{\text {Sob }}$ around the median is 0.26 dex (i.e. $68 \%$ of all cells lie within a factor of $\sim 1.8$ of the median).

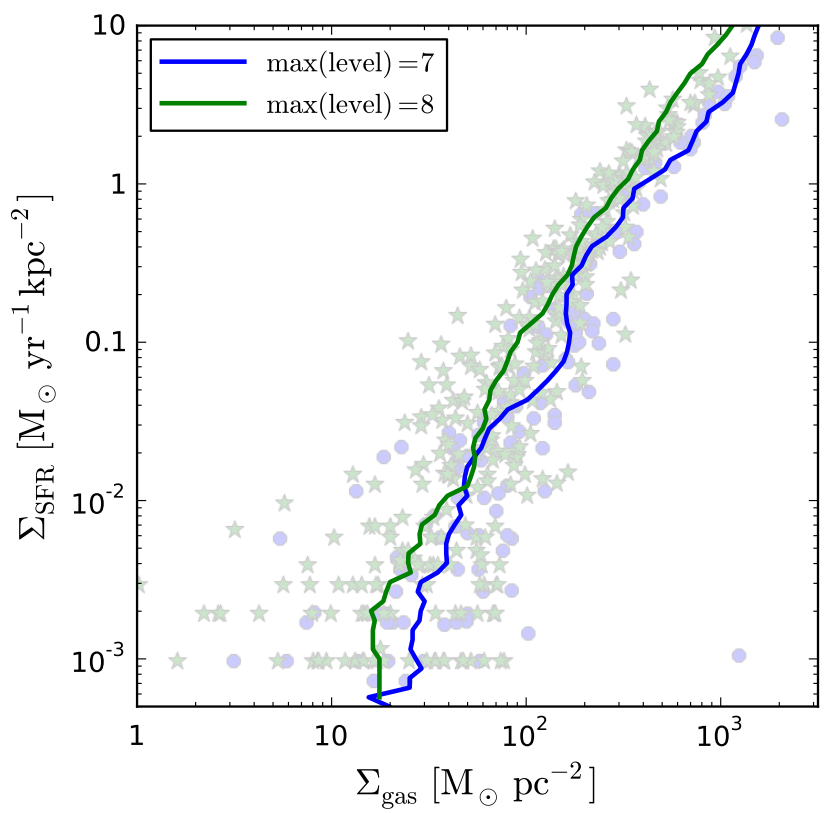

Figure 22. Comparison of the KS relation for the KMT09_Sob and KMT09_SobL8 simulations at $z=6$. The use of $\Sigma_{\text {Sob }}$ does not fully remove the resolution dependence, but reduces it mildly compared to simulations with $\Sigma_{\text {cell }}$ (cf. right panel of Fig. 10].

This post-processing analysis indicates that a simulation actually using the Sobolev-like approximation at run-time might have somewhat reduced SF, owing to the systematically slightly lower columns, which provide less shielding and hence lower $\mathrm{H}_{2}$ abundances. This is in fact borne out in practice, as shown in the right panel of Fig. 21. We ran two additional simulations, KMT09_Sob and KMT09_SobL8, in which the column densities entering the KMT09 $f_{\mathrm{H}_{2}}$ prescription were determined at run-time using the Sobolev-like estimator. Indeed the resulting KS relation has a slightly lower normalization, indicating a somewhat reduced SF efficiency.

As discussed in the main text, to some degree there will always be a resolution dependence in the determination of the column density in our simulations, simply because we are not resolving the true Jeans length and are applying a minimum pressure support in order to prevent artificial fragmentation. This is demonstrated in Fig. 22, which shows the KS relations for the KMT09_Sob and KMT09_SobL8 simulations. The corresponding plot for simulations with $\Sigma_{\text {cell }}$ is the right panel of Fig. 10, and a comparison between these two figures shows that the use of $\Sigma_{\text {Sob }}$ only mildly reduces the resolution dependence.

The two different methods of estimating column densities $\left(\Sigma_{\text {cell }}, \Sigma_{\text {Sob }}\right)$ are resolution-dependent in different ways. The difference between the two in $\Sigma$ and in the KS relation is fairly small (less than a factor of 2), and does not impact the overall conclusion of our study that the metallicity-dependent nature of the atomic-to-molecular transition can play a major role in explaining the low star formation efficiency in dwarf galaxies. 